\title{
Deformations of Maxwell Superalgebras and Their Applications
}

\author{
Sotirios Bonanos ${ }^{1}$, Joaquim Gomis ${ }^{2}$, Kiyoshi Kamimura ${ }^{3}$ and Jerzy Lukierski ${ }^{4}$ \\ ${ }^{1}$ Institute of Nuclear Physics, NCSR Demokritos, 15310 Aghia Paraskevi, Attiki, Greece \\ ${ }^{2}$ Departament ECM and ICCUB, Universitat de Barcelona, Diagonal 647, 08028 \\ Barcelona, Spain \\ ${ }^{3}$ Department of Physics, Toho University, Funabashi, 274-8510 Japan \\ ${ }^{4}$ Institute of Theoretical Physics, Wroclaw University, pl. Maxa Borna 9, 50-204 \\ Wroclaw, Poland
}

\begin{abstract}
We describe the Lie algebra deformations of $\mathrm{D}=4$ Maxwell superalgebra that was recently introduced as the symmetry algebra of a kappa-symmetric massless superparticle in a supersymmetric constant electromagnetic background. Further we introduce the $\mathrm{D}=3$ Maxwell superalgebra and present all its possible deformations. Finally the deformed superalgebras are used to derive via a contraction procedure the complete set of Casimir operators for $\mathrm{D}=4$ and $\mathrm{D}=3$ Maxwell superalgebras.
\end{abstract}




\section{Contents}

1. Introduction 2

2. $\mathrm{D}=4$ Maxwell superalgebra and its Weyl-enlargement

3. Deformations of Maxwell superalgebra in $\mathrm{D}=4$

$3.1 k$-deformation 8

3.1 .1 Anti-de Sitter case: $k>0$

3.1 .2 de Sitter case: $k<0$

$3.2 s$-deformation 11

4. $\mathrm{D}=3$ Maxwell superalgebra and its deformations 11

$4.1 \mathrm{D}=3$ Maxwell superalgebra 11

4.2 Deformations of $\mathrm{D}=3$ Maxwell superalgebra 12

4.2.1 $\quad k^{+}$-deformation

$4.2 .2 \quad k^{-}$-deformation 13

4.2.3 $c$-deformation 13

5. Casimir Operators of the Maxwell superalgebras 14

5.1 Casimir Operators of the Maxwell superalgebras in $\mathrm{D}=4$

5.1.1 Casimir operators of $\mathrm{O}(\mathrm{N})$ algebras 15

$5.1 .2 O S p(2 \mid 4)$ Casimir operators 15

5.1.3 Casimir operators of the Maxwell superalgebra by contraction 16

5.2 Casimir operators of $\mathrm{D}=3$ Maxwell superalgebras 18

6. Conclusions and Outlook 19

A. Notations and conventions 22

B. Quaternionic (super)groups and (super)algebras 22

B.1 Quaternionic algebras 22

B.2 Quaternionic groups and corresponding Lie algebras [32] [33] 22

B.3 Quaternionic supergroups and applications to space-time symmetries 24

B.4 $\mathrm{D}=4 \mathrm{dS}$ superalgebra 25 


\section{Introduction}

The Poincarè algebra and Poincarè group describe the symmetries of empty Minkowski space-time. Filling such a flat space-time with some background fields leads to a modification of Poincarè symmetries. An example of such a modification is the so-called Maxwell symmetries, which was obtained already in the seventies [1] 2] by considering Minkowski space with an added constant electromagnetic (EM) background. The collection of arbitrary values of the constant EM field strengths provides additional degrees of freedom in Minkowski space, supplementing the Poincarè group with additional group parameters and the Poincarè algebra with new generators.

The Maxwell algebra [2]-[13], see also [15]-16] for $\mathrm{D}=3$, is obtained by adding to the Poincarè generators $\left(P_{\mu}, M_{\mu \nu}\right)$ the tensorial central charges $Z_{\mu \nu}\left(Z_{\mu \nu}=-Z_{\nu \mu}\right)$ which modify the commutativity of the four-momenta $P_{\mu}$

$$
\left[P_{\mu}, P_{\nu}\right]=i Z_{\mu \nu}
$$

where $M_{\mu \nu}$ are the Lorentz algebra generators and

$$
\begin{aligned}
{\left[Z_{\mu \nu}, M_{\rho \sigma}\right] } & =-i \eta_{\nu[\rho} Z_{|\mu| \sigma]}+i \eta_{\mu[\rho} Z_{|\nu| \sigma]} \\
{\left[P_{\mu}, Z_{\rho \sigma}\right] } & =\left[Z_{\mu \nu}, Z_{\rho \sigma}\right]=0
\end{aligned}
$$

The D-dimensional Maxwell algebra $G=\left(M_{\mu \nu}, P_{\mu}, Z_{\mu \nu}\right)$ has the structure of a semi-direct sum

$$
G=O(D-1,1) \boxplus H
$$

where the algebra $H\left(=\left(P_{\mu}, Z_{\mu \nu}\right)\right)$ can be obtained by suitable contraction $\alpha \rightarrow 0$ of the de Sitter algebra $O(D, 1)=\left(\mathcal{M}_{\mu \nu}, \mathcal{P}_{\mu}\right)$, or anti-de Sitter $O(D-1,2)$, with [10]

$$
\mathcal{M}_{\mu \nu}=\frac{1}{\alpha^{2}} Z_{\mu \nu}, \quad \mathcal{P}_{\mu}=\frac{1}{\alpha} P_{\mu} .
$$

The Maxwell algebras and Maxwell symmetries were recently studied in three different directions:

1. The Maxwell algebra is an enlargement of Poincarè algebra, i.e. by putting $Z_{\mu \nu}=0$ one gets back to the Poincarè algebra. Analogously, one can consider the class of supersymmetrizations providing Maxwell superalgebra as the minimal enlargement of $\mathrm{N}=1$ Poincarè superalgebra. Such supersymmetric extension of $\mathrm{D}=4$ Maxwell algebra was obtained in [10] by adding minimal number of two four-dimensional Majorana supercharges $\mathbf{Q}_{\alpha}, \boldsymbol{\Sigma}_{\alpha}$ and mathematically optional two scalar generators $B_{5}, B$. The coset $\frac{\text { SuperMaxwell }}{\text { Lorent } x \times B_{5}}$ describes the supersymmetries of flat (Wess-Zumino) Minkowski superspace with arbitrary constant values of an Abelian gauge superfield background

$$
W_{\alpha}(\theta)=i \lambda_{\alpha}-\frac{i}{2} f_{\mu \nu}\left(\bar{\theta} \gamma^{\mu \nu}\right)_{\alpha}-i D\left(\bar{\theta} \gamma_{5}\right)_{\alpha} .
$$

The superspace coordinates $\left(x^{\mu}, \theta^{\alpha}, \phi\right)$ are supplemented in the framework of Maxwell supergeometry by graded additional coordinates $\left(\lambda_{\alpha}, f_{\mu \nu}, D\right)$ related to the generators $\left(\Sigma_{\alpha}, Z_{\mu \nu}, B\right)$. 
2. Following preliminary results obtained in [5], all deformations of the Maxwell algebra in any dimension $\mathrm{D}=\mathrm{d}+1$ were studied recently in $[8]$. In arbitrary dimension $\mathrm{D}$ there is a "universal" $k$-deformation, resulting in the following deformed Maxwell algebras:

$$
\begin{array}{lr}
k>0: O(d, 1) \oplus O(d, 2) \quad(\text { Lorent } \oplus \oplus A d S) \\
k<0: O(d, 1) \oplus O(d+1,1) \quad(\text { Lorentz } \oplus d S)
\end{array}
$$

In $\mathrm{D}=3$ the deformations are parametrized by two parameters $(k, b)$, with an additional "exotic" $b$-deformation. The $(k, b)$ plane can be divided into two domains where the two deformed Maxwell algebras described by (1.7) are realized. However on the curve separating these two domains the obtained algebra is isomorphic to $O(2,1) \oplus I S O(2,1)(\mathrm{D}=3$ Lorentz $\oplus \mathrm{D}=3$ Poincarè $)$.

3. One can study further extensions of the Poincarè symmetries by adding new tensorial central generators [6] [7] to the first level extension described by the Maxwell algebra. For example, in $\mathrm{D}=4$ the second level extension consists in adding the third rank tensorial charges $Y_{\mu[\rho \sigma]}$, which can be related to a Minkowski space filled with arbitrary linear EM background (i.e., $F_{[\mu \nu]}=f_{[\mu \nu]}+f_{[\mu \nu] \rho} x^{\rho}, f_{[\mu \nu]}$ and $f_{[\mu \nu] \rho}$ arbitrary constant tensors).

The first aim of this paper is to consider all possible deformations of the $\mathrm{D}=4$ Maxwell superalgebra introduced in [10]. In $\mathrm{D}=4$ one obtains two independent deformations:

- First, the supersymmetrization of the "universal" $k$-deformation given by (1.7). Because of the doubling of Majorana supercharges in the Maxwell superalgebra, the deformed superalgebras in $\mathrm{D}=4$ require also eight real supercharges describing $\mathrm{N}=2$ AdS and $\mathrm{N}=1 \mathrm{dS}$ SUSY ${ }^{1}$

$$
\begin{aligned}
& k>0: O(3,1) \oplus O S p(2 \mid 4) \oplus R \\
& k<0: O(3,1) \oplus U U_{\alpha}(1,1 \mid 1 ; H) \oplus R
\end{aligned}
$$

where $U U_{\alpha}(1,1 \mid 1 ; H)$ is $\mathrm{N}=1, \mathrm{D}=4$ de-Sitter superalgebra [17] 18] and will be explained in detail in Appendix B.

- Second, the $s$-deformation which does not have a non-SUSY counterpart. It involves only a modification of the algebraic relations for the scalar generator $B_{5}$ with the dilatation operator $\mathcal{D}$ given by the replacement $B_{5} \rightarrow B_{5}+s \mathcal{D}$. If we enlarge the Maxwell superalgebra by Weyl symmetry then the $s$-deformation is no longer an independent deformation ${ }^{2}$.

Second aim of the paper is to introduce the Maxwell superalgebra in $\mathrm{D}=3$ and study its possible deformations. The additional $s$-deformation is not present in $\mathrm{D}=3$. We find that

\footnotetext{
${ }^{1}$ For the simplest $\mathrm{N}=1$ supersymmetrization of $\mathrm{D}=4$ de-Sitter algebra we need 8 supercharges (see Appendix B)

${ }^{2} \mathrm{An}$ analogous situation appears in the deformations of the symmetries of very special relativity [20].
} 
there is a two parameter deformation of $\mathrm{D}=3$ Maxwell superalgebra as in the bosonic case considered in [8]. Depending on the values of the deformation parameters, we find three different deformed superalgebras: $O(2,1) \oplus O S p(1 \mid 2) \oplus O S p(1 \mid 2), O(2,1) \oplus O S p(1 \mid 2 ; C) \oplus$ $\overline{O S p(1 \mid 2 ; C)}$ and $O S p(1 \mid 2) \oplus(\mathrm{D}=3$ superPoincarè $)$.

The plan of the paper is the following: In section 2 we recall the results on $\mathrm{D}=4$ Maxwell superalgebra [10] and introduce its one-dimensional Weyl extension by adding appropriate scale transformations ${ }^{3}$. It appears that the superMaxwell-invariant massless superparticle model, introduced in [10], is also invariant under the Maxwell-Weyl supersymmetry. In section 3 we discuss two deformations of the $\mathrm{D}=4$ Maxwell superalgebra. One ( $k$-deformation) is described by the superalgebras (1.8) and (1.9), and the other $(s$ deformation) can be introduced as a parameter-dependent class of subalgebras of the $\mathrm{D}=4$ Maxwell-Weyl superalgebra. In section 4 we introduce the $\mathrm{D}=3$ Maxwell superalgebra and obtain the supersymmetrization of the two-parameter family of deformations.

In [10] we have presented the bilinear Casimir operators of the $\mathrm{D}=4$ Maxwell superalgebra, including the generalized mass-shell formula. In section 5 , by contracting the known Casimir operators of (1.8) $(k \rightarrow 0)$ we obtain all six Casimirs of the $\mathrm{D}=4$ Maxwell superalgebra. Subsequently, the Casimir operators for $\mathrm{D}=3$ Maxwell superalgebra are also obtained via contraction. In section 6 we present conjectures about the existence of Maxwell superalgebras for $D>4$ and its deformations, outline the relation with other proposals [21] 22] and conclude with some final remarks. We add also two appendices, one summarizing our conventions including gamma matrices, and a second describing quaternionic (super) groups and (super) algebras as well as the $\mathrm{D}=4, \mathrm{~N}=1$ de-Sitter superalgebra $U U_{\alpha}(1,1 \mid 1 ; H)$ appearing in (1.9).

\section{2. $\mathrm{D}=4$ Maxwell superalgebra and its Weyl-enlargement}

In a recent paper [10] we have proposed the following supersymmetric extension, denoted by $\mathcal{G}_{5}$, of the Maxwell algebra in 4 dimensions (our notations and conventions are summarized in Appendix A),

$$
\begin{array}{rlrl}
{\left[P_{\mu}, P_{\nu}\right]} & =i Z_{\mu \nu}, & {\left[P_{\mu}, \mathbf{Q}_{\alpha}\right]=-i \boldsymbol{\Sigma}_{\beta}\left(\gamma_{\mu}\right)_{\alpha}^{\beta},} \\
\left\{\mathbf{Q}_{\alpha}, \mathbf{Q}_{\beta}\right\} & =2\left(C \gamma^{\mu}\right)_{\alpha \beta} P_{\mu}, \quad\left\{\mathbf{Q}_{\alpha}, \boldsymbol{\Sigma}_{\beta}\right\}=\frac{1}{2}\left(C \gamma^{\mu \nu}\right)_{\alpha \beta} Z_{\mu \nu}+\left(C \gamma_{5}\right)_{\alpha \beta} B, \\
{\left[B_{5}, \mathbf{Q}_{\alpha}\right]} & =-i\left(\mathbf{Q} \gamma_{5}\right)_{\alpha}, \quad\left[B_{5}, \boldsymbol{\Sigma}_{\alpha}\right]=i\left(\boldsymbol{\Sigma} \gamma_{5}\right)_{\alpha}, \\
{\left[P_{\mu}, M_{\rho \sigma}\right]} & =-i \eta_{\mu[\rho} P_{\sigma]}, \quad\left[Z_{\mu \nu}, M_{\rho \sigma}\right]=-i \eta_{\nu[\rho} Z_{|\mu| \sigma]}+i \eta_{\mu[\rho} Z_{|\nu| \sigma]}, \\
{\left[M_{\rho \sigma}, \mathbf{Q}_{\alpha}\right]} & =-\frac{i}{2}\left(\mathbf{Q} \gamma_{\rho \sigma}\right)_{\alpha}, \quad\left[M_{\rho \sigma}, \boldsymbol{\Sigma}_{\alpha}\right]=-\frac{i}{2}\left(\boldsymbol{\Sigma} \gamma_{\rho \sigma}\right)_{\alpha}, \\
{\left[M_{\mu \nu}, M_{\rho \sigma}\right]} & =-i \eta_{\nu[\rho} M_{|\mu| \sigma]}+i \eta_{\mu[\rho} M_{|\nu| \sigma]} .
\end{array}
$$

The bosonic generators $\left(P_{\mu}, M_{\mu \nu}, Z_{\mu \nu}\right)$, linked to translations, Lorentz rotations and additional tensorial coordinates, form the bosonic Maxwell subalgebra and the fermionic generators $\mathbf{Q}_{\alpha}, \boldsymbol{\Sigma}_{\alpha},(\alpha=1,2,3,4)$ are two Majorana spinor charges. $B$ is a central charge

\footnotetext{
${ }^{3}$ One could also consider the enlargement of pure bosonic Maxwell algebra by dilatations.
} 
and $B_{5}$ generates chiral transformations. We point out that $\mathrm{D}=4$ Maxwell superalgebra can also be considered as an enlargement by generators $Z_{\mu \nu}$ of the algebra with 8 supercharges introduced by Green 23.

There are three subalgebras obtained by consistently removing generators $B$ and/or $B_{5}$ from (2.1) (see 10]).

1) The minimal supersymmetric extension $\mathcal{G}$, with a bosonic sector consisting only of the Maxwell algebra generators, is obtained if we remove $B$ and $B_{5}$.

2) Removing only generator $B_{5}$, we get a central extension $\tilde{\mathcal{G}}$ of $\mathcal{G}$. The generator $B$ is required if we wish to introduce the scalar degree of freedom describing the off-shell extension of $\mathrm{D}=4 U(1)$ field strength supermultiplet.

3) One can consider a subalgebra with only the generator $B_{5}$ which acts on the supercharges $\mathbf{Q}_{\alpha}, \boldsymbol{\Sigma}_{\alpha}$ as chiral generator. If $B$ is present, $B_{5}$ is also required for the existence of the supersymmetric mass Casimir.

In this paper we shall consider the Maxwell superalgebra $\mathcal{G}_{5}$ with both $B$ and $B_{5}$ given in (2.1). We add that all cases describe the supersymmetric extension of the Maxwell algebra with minimal number of supercharges (eight real or four complex) and all these supersymmetrizations describe $\mathrm{N}=1$ Maxwell superalgebra ${ }^{4}$. We note that four additional supercharges $\boldsymbol{\Sigma}_{\alpha}$ are present due to the supersymmetrization of the constant electromagnetic background (1.6).

The superalgebra $\mathcal{G}_{5}$ describes the symmetries of the massless kappa invariant superparticle action in an external constant $\mathrm{N}=1$ susy invariant background presented in [10]

$$
\mathcal{L}=\frac{\pi_{\mu}^{2}}{2 e}+\frac{1}{2} f_{\mu \nu} L_{Z}^{\mu \nu}+i \lambda_{\alpha} L_{\Sigma}^{\alpha}+D L_{B}
$$

where $L_{P}^{\mu}=\pi^{\mu}=d x^{\mu}+i \bar{\theta} \gamma^{\mu} \dot{\theta}, L_{Z}^{\mu \nu}, L_{\Sigma}^{\alpha}, L_{B}$ are the pullbacks on the world line of the components of the MC forms $\Omega=-i g^{-1} d g$ defined on the supercoset $\mathcal{G}_{5} /\left(\right.$ Lorent $\left.z \otimes B_{5}\right)$

$$
g=e^{\frac{i}{2} Z_{\mu \nu} \phi^{\mu \nu}} e^{i P_{\mu} x^{\mu}} e^{i \boldsymbol{\Sigma}_{\alpha} \phi^{\alpha}} e^{i \mathbf{Q}_{\alpha} \theta^{\alpha}} e^{i B \phi} .
$$

We observe that one can assign mass dimensions to the generators of the superalgebra (2.1) as follows

$$
\left[P_{\mu}\right]=1, \quad\left[Z_{\mu \nu}\right]=2, \quad\left[\mathbf{Q}_{\alpha}\right]=\frac{1}{2}, \quad\left[\boldsymbol{\Sigma}_{\alpha}\right]=\frac{3}{2}, \quad[B]=2, \quad\left[B_{5}\right]=\left[M_{\mu \nu}\right]=0,
$$

which can be described by introducing a dilatation generator $\mathcal{D}$ satisfying the relations:

$$
\begin{aligned}
& {\left[\mathcal{D}, P_{\mu}\right]=i P_{\mu}, \quad\left[\mathcal{D}, Z_{\mu \nu}\right]=2 i Z_{\mu \nu}, \quad\left[\mathcal{D}, \mathbf{Q}_{\alpha}\right]=\frac{i}{2} \mathbf{Q}_{\alpha},} \\
& {\left[\mathcal{D}, \boldsymbol{\Sigma}_{\alpha}\right]=\frac{3}{2} i \boldsymbol{\Sigma}_{\alpha}, \quad[\mathcal{D}, B]=2 i, \quad\left[\mathcal{D}, B_{5}\right]=\left[\mathcal{D}, M_{\mu \nu}\right]=0 .}
\end{aligned}
$$

The supercoset coordinates in (2.3) transform under the scale transformations, generated by $\mathcal{D}$, with opposite mass dimensionalities:

$$
x^{\mu^{\prime}}=\lambda^{-1} x^{\mu}, \quad \phi^{\mu \nu^{\prime}}=\lambda^{-2} \phi^{\mu \nu}, \quad \theta^{\alpha^{\prime}}=\lambda^{-1 / 2} \theta^{\alpha}, \quad \phi^{\alpha^{\prime}}=\lambda^{-3 / 2} \phi^{\alpha}, \quad \phi^{\prime}=\lambda^{-2} \phi .
$$

\footnotetext{
${ }^{4}$ We mention that the superalgebra $O(3,1) \oplus O S p(1 \mid 4)$ with four real supercharges considered in 11$] 22$ describe the supersymmetrization of one of the deformations of Maxwell superalgebra (see also section 6).
} 
Adding relations (2.5) to (2.1) one obtains the Maxwell-Weyl superalgebra, which is a one-dimensional enlargement of Maxwell superalgebra described by the semidirect sum $\mathcal{D} \rightrightarrows \mathcal{G}_{5}$. We note that the massless superparticle action (2.2) remains invariant under the scale transformation with the einbein, transforming as $e^{\prime}=\lambda^{-2} e$, consistent with its role as a coordinate for $\mathcal{D}$ in the coset $\mathcal{D} \rightrightarrows \mathcal{G}_{5} /\left(\right.$ Lorent $\left.z \otimes B_{5}\right)$.

\section{Deformations of Maxwell superalgebra in $\mathrm{D}=4$}

The Maxwell superalgebra (2.1) is equivalently described in terms of the Maurer Cartan one form

$$
\Omega=P_{\mu} L_{P}^{\mu}+\frac{1}{2} M_{\mu \nu} L_{M}^{\mu \nu}+\frac{1}{2} Z_{\mu \nu} L_{Z}^{\mu \nu}+B L_{B}+B_{5} L^{5}+\mathbf{Q}_{\alpha} \mathbf{L}^{\alpha}+\mathbf{\Sigma}_{\alpha} \mathbf{L}_{\Sigma}^{\alpha},
$$

satisfying the Maurer Cartan equation $d \Omega+i \Omega \wedge \Omega=0$, as

$$
\begin{aligned}
d L_{P}^{\mu}+L_{M}^{\mu \nu} L_{P \nu}-i \overline{\mathbf{L}} \gamma^{\mu} \mathbf{L} & =0 \\
d L_{M}^{\mu \nu}+L_{M}^{\mu \rho} \eta_{\rho \sigma} L_{M}^{\sigma \nu} & =0, \\
d L_{Z}^{\mu \nu}+L_{M}^{\mu \rho} \eta_{\rho \sigma} L_{Z}^{\sigma \nu}+L_{Z}^{\mu \rho} \eta_{\rho \sigma} L_{M}^{\sigma \nu}-L_{P}^{\mu} L_{P}^{\nu}-i \overline{\mathbf{L}} \gamma^{\mu \nu} \mathbf{L}_{\Sigma} & =0 \\
d \mathbf{L}^{\alpha}+\frac{1}{4} L_{M}^{\mu \nu}\left(\gamma_{\mu \nu} \mathbf{L}\right)^{\alpha}+L^{5}\left(\gamma_{5} \mathbf{L}\right)^{\alpha} & =0 \\
d \mathbf{L}_{\Sigma}^{\alpha}+\frac{1}{4} L_{M}^{\mu \nu}\left(\gamma_{\mu \nu} \mathbf{L}_{\Sigma}\right)^{\alpha}+L_{P}^{\mu}\left(\gamma_{\mu} \mathbf{L}\right)^{\alpha}-L^{5}\left(\gamma_{5} \mathbf{L}_{\Sigma}\right)^{\alpha} & =0 \\
d L_{B}-i \overline{\mathbf{L}} \gamma_{5} \mathbf{L}_{\Sigma} & =0 \\
d L^{5} & =0
\end{aligned}
$$

These MC equations provide a dual formulation of the Maxwell superalgebra (2.1) and closure of the system (3.2) under exterior differentiation is equivalent to the Jacobi identities of the algebra being satisfied. The deformations of the algebra can be studied using cohomological methods [24, see also for example [20]. A non-trivial deformation is obtained if it is possible to add covariantly closed but not covariantly exact two forms to the right-hand-sides of the MC equations (3.2). Covariant exterior differentiation is defined here in terms of the connection 1-forms $\omega^{A}{ }_{B}=C^{A}{ }_{B C} L^{C}$, where $C^{A}{ }_{B C}$ are the structure constants and $L^{C}$ the MC 1-forms of the undeformed algebra. The Jacobi identities imply that the connection is flat: $d \omega^{A}{ }_{B}+\omega^{A}{ }_{C} \wedge \omega^{C}{ }_{B}=0$.

A systematic examination ${ }^{5}$ yields two possible non-trivial deformations in 4 dimensions, up to redefinitions using covariantly exact one forms. First one is the $k$-deformation, a supersymmetric extension of the $k$-deformation of the bosonic Maxwell algebra in [8],

$$
\begin{aligned}
d L_{P}^{\mu}+L_{M}^{\mu \nu} L_{P \nu}-i \overline{\mathbf{L}} \gamma^{\mu} \mathbf{L} & =k\left(L_{Z}^{\mu \nu} L_{P \nu}+\frac{i}{4} \overline{\mathbf{L}_{\Sigma}} \gamma^{\mu} \mathbf{L}_{\Sigma}\right), \\
d L_{M}^{\mu \nu}+L_{M}^{\mu \rho} \eta_{\rho \sigma} L_{M}^{\sigma \nu} & =0 \\
d L_{Z}^{\mu \nu}+L_{M}^{\mu \rho} \eta_{\rho \sigma} L_{Z}^{\sigma \nu}+L_{Z}^{\mu \rho} \eta_{\rho \sigma} L_{M}^{\sigma \nu}-L_{P}^{\mu} L_{P}^{\nu}-i \overline{\mathbf{L}} \gamma^{\mu \nu} \mathbf{L}_{\Sigma} & =k L_{Z}^{\mu \rho} L_{Z \rho}{ }^{\nu}
\end{aligned}
$$

\footnotetext{
${ }^{5}$ Some of the calculations with forms were done using the Mathematica code for differential forms developed by S. Bonanos. See: "Graded Exterior Differential Calculus" 25].
} 


$$
\begin{aligned}
d \mathbf{L}^{\alpha}+\frac{1}{4} L_{M}^{\mu \nu}\left(\gamma_{\mu \nu} \mathbf{L}\right)^{\alpha}+L^{5}\left(\gamma_{5} \mathbf{L}\right)^{\alpha} & =\frac{k}{4}\left(L_{Z}^{\mu \nu}\left(\gamma_{\mu \nu} \mathbf{L}\right)^{\alpha}-L_{P}^{\mu}\left(\gamma_{\mu} \mathbf{L}_{\Sigma}\right)^{\alpha}\right) \\
d \mathbf{L}_{\Sigma}^{\alpha}+\frac{1}{4} L_{M}^{\mu \nu}\left(\gamma_{\mu \nu} \mathbf{L}_{\Sigma}\right)^{\alpha}+L_{P}^{\mu}\left(\gamma_{\mu} \mathbf{L}\right)^{\alpha}-L^{5}\left(\gamma_{5} \mathbf{L}_{\Sigma}\right)^{\alpha} & =\frac{k}{4} L_{Z}^{\mu \nu}\left(\gamma_{\mu \nu} \mathbf{L}_{\Sigma}\right)^{\alpha} \\
d L_{B}-i \overline{\mathbf{L}} \gamma_{5} \mathbf{L}_{\Sigma} & =0 \\
d L^{5} & =-k i \overline{\mathbf{L}} \gamma_{5} \mathbf{L}_{\Sigma}
\end{aligned}
$$

where $k$ is the deformation parameter having the mass dimension $[k]=2$. Note that in contrast to (3.2) we cannot have the closed algebra without $L^{5}$ (chiral symmetry) in the deformed algebra (3.3). In other words we cannot contract out $L^{5}$ by $L^{5} \rightarrow a L^{5}$ and $a \rightarrow 0$ in the last equation of (3.3). The closed MC equation (3.3) is written in the form of a superalgebra,

$$
\begin{aligned}
{\left[P_{\mu}, P_{\nu}\right] } & =i Z_{\mu \nu}, \quad\left[P_{\mu}, Z_{\rho \sigma}\right]=i k \eta_{\mu[\rho} P_{\sigma]}, \\
{\left[Z_{\mu \nu}, Z_{\rho \sigma}\right] } & =i k\left(\eta_{\nu[\rho} Z_{|\mu| \sigma]}-\eta_{\mu[\rho} Z_{|\nu| \sigma]}\right), \\
{\left[P_{\mu}, M_{\rho \sigma}\right] } & =-i \eta_{\mu[\rho} P_{\sigma]}, \quad\left[Z_{\mu \nu}, M_{\rho \sigma}\right]=-i \eta_{\nu[\rho} Z_{|\mu| \sigma]}+i \eta_{\mu[\rho} Z_{|\nu| \sigma]}, \\
{\left[M_{\mu \nu}, M_{\rho \sigma}\right] } & =-i \eta_{\nu[\rho} M_{|\mu| \sigma]}+i \eta_{\mu[\rho} M_{|\nu| \sigma]}, \\
\left\{\mathbf{Q}_{\alpha}, \mathbf{Q}_{\beta}\right\} & =2\left(C \gamma^{\mu}\right)_{\alpha \beta} P_{\mu}, \quad\left\{\boldsymbol{\Sigma}_{\alpha}, \boldsymbol{\Sigma}_{\beta}\right\}=\frac{1}{2} k\left(C \gamma^{\mu}\right)_{\alpha \beta} P_{\mu}, \\
\left\{\mathbf{Q}_{\alpha}, \mathbf{\Sigma}_{\beta}\right\} & =\frac{1}{2}\left(C \gamma^{\mu \nu}\right)_{\alpha \beta} Z_{\mu \nu}+\left(C \gamma_{5}\right)_{\alpha \beta}\left(B-k B_{5}\right), \\
{\left[P_{\mu}, \mathbf{Q}_{\alpha}\right] } & =-i \boldsymbol{\Sigma}_{\beta}\left(\gamma_{\mu}\right)_{\alpha}, \quad\left[P_{\mu}, \boldsymbol{\Sigma}_{\alpha}\right]=-\frac{i}{4} k \mathbf{Q}_{\beta}\left(\gamma_{\mu}\right)_{\alpha}^{\beta}, \\
{\left[Z_{\mu \nu}, \mathbf{Q}_{\alpha}\right] } & =\frac{i}{2} k\left(\mathbf{Q} \gamma_{\mu \nu}\right)_{\alpha}, \quad\left[Z_{\mu \nu}, \boldsymbol{\Sigma}_{\alpha}\right]=\frac{i}{2} k\left(\boldsymbol{\Sigma} \gamma_{\mu \nu}\right)_{\alpha}, \\
{\left[B_{5}, \mathbf{Q}_{\alpha}\right] } & =-i\left(\mathbf{Q} \gamma_{5}\right)_{\alpha}, \quad\left[B_{5}, \boldsymbol{\Sigma}_{\alpha}\right]=i\left(\boldsymbol{\Sigma} \gamma_{5}\right)_{\alpha}, \\
{\left[M_{\rho \sigma}, \mathbf{Q}_{\alpha}\right] } & =-\frac{i}{2}\left(\mathbf{Q} \gamma_{\rho \sigma}\right)_{\alpha}, \quad\left[M_{\rho \sigma}, \boldsymbol{\Sigma}_{\alpha}\right]=-\frac{i}{2}\left(\boldsymbol{\Sigma} \gamma_{\rho \sigma}\right)_{\alpha} .
\end{aligned}
$$

The second $s$-deformation is

$$
\begin{aligned}
d L_{P}^{\mu}+L_{M}^{\mu \nu} L_{P \nu}-i \overline{\mathbf{L}} \gamma^{\mu} \mathbf{L} & =s L^{5} L_{P}^{\mu}, \\
d L_{M}^{\mu \nu}+L_{M}^{\mu \rho} \eta_{\rho \sigma} L_{M}^{\sigma \nu} & =0 \\
d L_{Z}^{\mu \nu}+L_{M}^{\mu \rho} \eta_{\rho \sigma} L_{Z}^{\sigma \nu}+L_{Z}^{\mu \rho} \eta_{\rho \sigma} L_{M}^{\sigma \nu}-L_{P}^{\mu} L_{P}^{\nu}-i \overline{\mathbf{L}} \gamma^{\mu \nu} \mathbf{L}_{\Sigma} & =2 s L^{5} L_{Z}^{\mu \nu}, \\
d \mathbf{L}^{\alpha}+\frac{1}{4} L_{M}^{\mu \nu}\left(\gamma_{\mu \nu} \mathbf{L}\right)^{\alpha}+L^{5}\left(\gamma_{5} \mathbf{L}\right)^{\alpha} & =\frac{1}{2} s L^{5} \mathbf{L}^{\alpha} \\
d \mathbf{L}_{\Sigma}^{\alpha}+\frac{1}{4} L_{M}^{\mu \nu}\left(\gamma_{\mu \nu} \mathbf{L}_{\Sigma}\right)^{\alpha}+L_{P}^{\mu}\left(\gamma_{\mu} \mathbf{L}\right)^{\alpha}-L^{5}\left(\gamma_{5} \mathbf{L}_{\Sigma}\right)^{\alpha} & =\frac{3}{2} s L^{5} \mathbf{L}_{\Sigma}^{\alpha}, \\
d L_{B}-i \overline{\mathbf{L}} \gamma_{5} \mathbf{L}_{\Sigma} & =2 s L^{5} L_{B}, \\
d L^{5} & =0 .
\end{aligned}
$$

where $s$ is the dimensionless deformation parameter. 


\section{$3.1 k$-deformation}

We describe the $k$-deformation of the Maxwell superalgebra in terms of known super algebras. We write

$$
L_{M}-k L_{Z}=L_{\mathcal{M}}, \quad L_{M}=L_{\mathcal{J}}, \quad L_{B}+\frac{1}{k} L^{5}=\frac{1}{k} L_{\mathcal{B}}, \quad L^{5}=L_{\mathcal{B}^{5}},
$$

and rescale using $k= \pm \frac{1}{R^{2}},(R>0)$,

$$
L_{P}^{\mu}=R L_{\mathcal{P}}^{\mu}, \quad \mathbf{L}^{\alpha}=\sqrt{R} \tilde{\mathbf{L}}^{\alpha}, \quad \mathbf{L}_{\Sigma}^{\alpha}=\sqrt{R^{3}} \tilde{\mathbf{L}}_{\Sigma}^{\alpha}
$$

so that all one forms are dimensionless. Correspondingly the relations of the new generators to those of $k$-deformed Maxwell superalgebra are found by comparing (3.1) with

$$
\Omega=\mathcal{P}_{\mu} L_{\mathcal{P}}^{\mu}+\frac{1}{2} \mathcal{M}_{\mu \nu} L_{\mathcal{M}}^{\mu \nu}+\frac{1}{2} \mathcal{J}_{\mu \nu} L_{\mathcal{J}}^{\mu \nu}+\mathcal{B} L_{\mathcal{B}}+\mathcal{B}_{5} L_{\mathcal{B}^{5}}+\tilde{\mathbf{Q}}_{\alpha} \tilde{\mathbf{L}}^{\alpha}+\tilde{\boldsymbol{\Sigma}}_{\alpha} \tilde{\mathbf{L}}_{\Sigma}^{\alpha}
$$

where

$$
\begin{aligned}
M_{\mu \nu} & =\mathcal{M}_{\mu \nu}+\mathcal{J}_{\mu \nu}, \quad Z_{\mu \nu}=\mp \frac{1}{R^{2}} \mathcal{M}_{\mu \nu}, \quad B= \pm \frac{1}{R^{2}} \mathcal{B}, \quad B_{5}=\mathcal{B}_{5}+\mathcal{B}, \\
P_{\mu} & =\frac{1}{R} \mathcal{P}_{\mu}, \quad \mathbf{Q}_{\alpha}=\frac{1}{\sqrt{R}} \tilde{\mathbf{Q}}_{\alpha}, \quad \boldsymbol{\Sigma}_{\alpha}=\frac{1}{\sqrt{R^{3}}} \tilde{\boldsymbol{\Sigma}}_{\alpha} .
\end{aligned}
$$

The MC equations (3.3) decompose to that of $O(3,1)$ of $\mathcal{J}$

$$
d L_{\mathcal{J}}^{\mu \nu}+L_{\mathcal{J}}^{\mu \rho} \eta_{\rho \sigma} L_{\mathcal{J}}^{\sigma \nu}=0
$$

the central charge $\mathcal{B}$,

$$
d L_{\mathcal{B}}=0
$$

and a superalgebra of $\left(\mathcal{P}, \mathcal{M}, \tilde{\mathbf{Q}}, \tilde{\boldsymbol{\Sigma}}, \mathcal{B}_{5}\right)$,

$$
\begin{aligned}
d L_{\mathcal{P}}^{\mu}+L_{\mathcal{M}}^{\mu \nu} L_{\mathcal{P} \nu}-i \overline{\tilde{\mathbf{L}}} \gamma^{\mu} \tilde{\mathbf{L}} & = \pm \frac{i}{4} \tilde{\mathbf{L}}_{\Sigma} \gamma^{\mu} \tilde{\mathbf{L}}_{\Sigma} \\
d L_{\mathcal{M}}^{\mu \nu}+L_{\mathcal{M}}^{\mu \rho} \eta_{\rho \sigma} L_{\mathcal{M}}^{\sigma \nu} & =\mp L_{\mathcal{P}}^{\mu} L_{\mathcal{P}}^{\nu} \mp i \overline{\tilde{\mathbf{L}}} \gamma^{\mu \nu} \tilde{\mathbf{L}}_{\Sigma}, \\
d \tilde{\mathbf{L}}^{\alpha}+\frac{1}{4} L_{\mathcal{M}}^{\mu \nu}\left(\gamma_{\mu \nu} \tilde{\mathbf{L}}\right)^{\alpha}+L_{\mathcal{B}^{5}}\left(\gamma_{5} \tilde{\mathbf{L}}\right)^{\alpha} & =\mp L_{\mathcal{P}}^{\mu} \frac{1}{4}\left(\gamma_{\mu} \tilde{\mathbf{L}}_{\Sigma}\right)^{\alpha} \\
d \tilde{\mathbf{L}}_{\Sigma}^{\alpha}+\frac{1}{4} L_{\mathcal{M}}^{\mu \nu}\left(\gamma_{\mu \nu} \tilde{\mathbf{L}}_{\Sigma}\right)^{\alpha}+L_{\mathcal{P}}^{\mu}\left(\gamma_{\mu} \tilde{\mathbf{L}}\right)^{\alpha}-L_{\mathcal{B}^{5}}\left(\gamma_{5} \tilde{\mathbf{L}}_{\Sigma}\right)^{\alpha} & =0 \\
d L_{\mathcal{B}^{5}} & =\mp i \tilde{\tilde{\mathbf{L}}} \gamma_{5} \tilde{\mathbf{L}}_{\Sigma}
\end{aligned}
$$

where upper signs correspond to $k>0$ and lower ones to $k<0$. Both cases $(k>0$ and $k<0$ ) will be discussed in detail below.

\subsubsection{Anti-de Sitter case: $k>0$}

We shall show that the superalgebra of $\left(\mathcal{P}, \mathcal{M}, \tilde{\mathbf{Q}}, \tilde{\boldsymbol{\Sigma}}, \mathcal{B}_{5}\right)$ in (3.14) for $k>0$ is isomorphic to $O S p(2 \mid 4)$, which is $\mathrm{N}=2, \mathrm{D}=4$ anti-de-Sitter superalgebra with bosonic subalgebras $O(2)$ 
and $S p(4) \sim O(3,2)$. The MC equation (3.14) for $k>0$ is written in $O(3,2)$ covariant form as

$$
\begin{aligned}
& d L_{\mathcal{M}}^{\hat{\mu} \hat{\nu}}+L_{\mathcal{M}}^{\hat{\mu} \hat{\rho}} \eta_{\hat{\rho} \hat{\sigma}} L_{\mathcal{M}}^{\hat{\sigma} \hat{\nu}}+\frac{i}{2} \overline{\mathbf{L}^{i}} \Gamma^{\hat{\mu} \hat{\nu}} \mathbf{L}^{i}=0, \\
& d \mathbf{L}^{\alpha i}+\frac{1}{4} L_{\mathcal{M}}^{\hat{\mu} \hat{\nu}}\left(\Gamma_{\hat{\mu} \hat{\nu}} \mathbf{L}\right)^{\alpha i}+L_{\mathcal{B}^{5}} \epsilon^{i j} \mathbf{L}^{\alpha j}=0 \\
& d L_{\mathcal{B}^{5}}-\frac{i}{2} \overline{\mathbf{L}}^{i} \epsilon^{i j} \mathbf{L}^{j}=0,
\end{aligned}
$$

where $\hat{\mu}, \hat{\nu}=0,1,2,3,4$ are $O(3,2)$ indices with the flat metric $\eta_{\hat{\mu} \hat{\nu}}=(-,+++,-)$ and $\epsilon^{12}=-\epsilon^{21}=1 . L_{\mathcal{M}}^{\mu \nu}$ 's are $O(3,1)$ components of $L_{\mathcal{M}}^{\hat{\mu} \hat{\nu}}$ and

$$
L_{\mathcal{P}}^{\mu}=L_{\mathcal{M}}^{\mu 4}
$$

We have also introduced $O(3,2)$ Majorana spinors

$$
\mathbf{L}^{i \alpha}=\left(\begin{array}{c}
\mathbf{L}^{1 \alpha} \\
\mathbf{L}^{2 \alpha}
\end{array}\right)=\left(\begin{array}{c}
\tilde{\mathbf{L}}^{\alpha}+\frac{1}{2} \tilde{\mathbf{L}}_{\Sigma}^{\alpha} \\
-\Gamma_{4}\left(\tilde{\mathbf{L}}^{\alpha}-\frac{1}{2} \tilde{\mathbf{L}}_{\Sigma}^{\alpha}\right)
\end{array}\right)
$$

and the $O(3,2)$ gamma matrices $\Gamma_{\hat{\mu}}$ given in appendix A. Recall that all gamma matrices are taken to be real in the Majorana representation. (3.15) shows that $\mathbf{L}^{i \alpha},(i=1,2)$ is a $O(2)$ doublet under rotations generated by $\mathcal{B}^{5}$. The dual superalgebra of the MC equation (3.15) is $\operatorname{OSp}(2 \mid 4)$ :

$$
\begin{aligned}
{\left[\mathcal{M}_{\hat{\mu} \hat{\nu}}, \mathcal{M}_{\hat{\rho} \hat{\sigma}}\right] } & =-i \eta_{\hat{\nu}[\hat{\rho}} \mathcal{M}_{|\hat{\mu}| \hat{\sigma}]}+i \eta_{\hat{\mu}[\hat{\rho}} \mathcal{M}_{|\hat{\nu}| \hat{\sigma}]}, \\
\left\{\mathcal{Q}_{\alpha i}, \mathcal{Q}_{\beta j}\right\} & =-\frac{1}{2} \delta_{i j}\left(C \Gamma^{\hat{\mu} \hat{\nu}}\right)_{\alpha \beta} \mathcal{M}_{\hat{\mu} \hat{\nu}}+\epsilon_{i j}(C)_{\alpha \beta} \mathcal{B}_{5}, \\
{\left[\mathcal{M}_{\hat{\mu} \hat{\nu}}, \mathcal{Q}_{\alpha i}\right] } & =-\frac{i}{2}\left(\mathcal{Q}_{i} \Gamma_{\hat{\mu} \hat{\nu}}\right)_{\alpha}, \\
{\left[\mathcal{B}_{5}, \mathcal{Q}_{\alpha i}\right] } & =i \epsilon_{i j} \mathcal{Q}_{\alpha j} .
\end{aligned}
$$

Thus the $k$-deformed Maxwell superalgebra for $k>0$ is isomorphic to $O S p(2 \mid 4) \oplus O(3,1) \oplus$ $U(1)$ with the generators related by (3.11),

$$
\begin{aligned}
& \mathcal{M}_{\mu 4}=R P_{\mu}, \quad \mathcal{M}_{\mu \nu}=-R^{2} Z_{\mu \nu}, \quad \mathcal{B}_{5}=B_{5}-R^{2} B \\
& \mathcal{Q}_{\alpha 1}=\frac{\sqrt{R}}{2} \mathbf{Q}_{\alpha}+\sqrt{R^{3}} \boldsymbol{\Sigma}_{\alpha}, \quad \mathcal{Q}_{\alpha 2}=\left(\frac{\sqrt{R}}{2} \mathbf{Q}_{\beta}-\sqrt{R^{3}} \boldsymbol{\Sigma}_{\beta}\right) \Gamma_{4 \alpha}^{\beta}, \\
& \mathcal{J}_{\mu \nu}=M_{\mu \nu}+R^{2} Z_{\mu \nu}, \quad \mathcal{B}=R^{2} B .
\end{aligned}
$$

The unconventional feature of the contraction (3.19) is exhibited in the formula for $\mathcal{J}_{\mu \nu}$ in which the direct sum structure of two Lorentz subalgebras belonging to $O S p(2 \mid 4) \oplus O(3,1)$ is not respected. Such nonstandard contractions generating nontrivial cohomologies and semidirect sum of algebras were considered in $\mathrm{D}=3$ (see [14] sect.8, [15]).

\subsection{2 de Sitter case: $k<0$}

The superalgebra of $\left(\mathcal{P}, \mathcal{M}, \tilde{\mathbf{Q}}, \tilde{\boldsymbol{\Sigma}}, \mathcal{B}_{5}\right)$ in 3.14 for $k=-\frac{1}{R^{2}}<0$ case is, as we will see, the $\mathrm{N}=1$ super dS algebra $U U_{\alpha}(1,1 \mid 1 ; H)$. In order to consider this case we introduce $O(4,1)$ metric and gamma matrices $\Gamma_{\hat{\mu}}$ as (see appendix A)

$$
\Gamma_{\mu}=\gamma_{\mu} \gamma_{5}, \quad \Gamma_{4}=i \gamma_{5}, \quad \eta_{\hat{\mu} \hat{\nu}}=(-,+++;+), \quad \hat{\mu}, \hat{\nu}=0,1,2,3,4 .
$$


In contrast to the AdS case these gamma matrices are not real but satisfy

$$
\Gamma_{\hat{\mu}}^{*}=-\Gamma_{4} \Gamma_{\hat{\mu}} \Gamma_{4}, \quad \Gamma_{\hat{\mu} \hat{\nu}}^{*}=\Gamma_{4} \Gamma_{\hat{\mu} \hat{\nu}} \Gamma_{4} .
$$

Using them we can rewrite (3.14) in $O(4,1)$ covariant form,

$$
\begin{aligned}
& d L_{\mathcal{M}}^{\hat{\mu} \hat{\nu}}+L_{\mathcal{M}}^{\hat{\mu} \hat{\rho}} \eta_{\hat{\rho} \hat{\sigma}} L_{\mathcal{M}}^{\hat{\sigma} \hat{\nu}}+\frac{1}{2} \overline{\mathbf{L}^{i}} \Gamma^{\hat{\mu} \hat{\nu}} \mathbf{L}^{i}=0 \\
& d \mathbf{L}^{\alpha i}+\frac{1}{4} L_{\mathcal{M}}^{\hat{\mu} \hat{\nu}}\left(\Gamma_{\hat{\mu} \hat{\nu}} \mathbf{L}\right)^{\alpha i}+L_{\mathcal{B}^{5}} \epsilon^{i j} \mathbf{L}^{\alpha j}=0 \\
& d L_{\mathcal{B}^{5}}-\frac{1}{2} \overline{\mathbf{L}}^{i} \epsilon^{i j} \mathbf{L}^{j}=0 .
\end{aligned}
$$

Here

$$
L_{\mathcal{M}}^{\mu 4}=L_{\mathcal{P}}^{\mu}, \quad \mathbf{L}^{i \alpha}=\left(\begin{array}{c}
\mathbf{L}^{1 \alpha} \\
\mathbf{L}^{2 \alpha}
\end{array}\right)=\left(\begin{array}{c}
\tilde{\mathbf{L}}^{\alpha}-\frac{i}{2} \tilde{\mathbf{L}}_{\Sigma}^{\alpha} \\
-i \Gamma_{4}\left(\tilde{\mathbf{L}}^{\alpha}+\frac{i}{2} \tilde{\mathbf{L}}_{\Sigma}^{\alpha}\right)
\end{array}\right)
$$

The $\mathbf{L}^{i \alpha}$ 's are not Majorana spinors but are symplectic Majorana spinors satisfying the condition, see for example 26],

$$
\mathbf{L}^{\alpha i \dagger}=i \epsilon^{i j}\left(\Gamma_{4}\right)_{\beta}^{\alpha} \mathbf{L}^{\beta j} .
$$

The symplectic Majorana spinors (3.23) transform as an $O(2)$ doublet under rotation by $\mathcal{B}^{5}$.

The dual superalgebra of the MC equation (3.22) is

$$
\begin{aligned}
{\left[\mathcal{M}_{\hat{\mu} \hat{\nu}}, \mathcal{M}_{\hat{\rho} \hat{\sigma}}\right] } & =-i \eta_{\hat{\nu}[\hat{\rho}} \mathcal{M}_{|\hat{\mu}| \hat{\sigma}]}+i \eta_{\hat{\mu}[\hat{\rho}} \mathcal{M}_{|\hat{\nu}| \hat{\sigma}]}, \\
\left\{\mathcal{Q}_{\alpha i}, \mathcal{Q}_{\beta j}\right\} & =\frac{i}{2} \delta_{i j}\left(C \Gamma^{\hat{\mu} \hat{\nu}}\right)_{\alpha \beta} \mathcal{M}_{\hat{\mu} \hat{\nu}}-i \epsilon_{i j}(C)_{\alpha \beta} \mathcal{B}_{5}, \\
{\left[\mathcal{M}_{\hat{\mu} \hat{\nu}}, \mathcal{Q}_{\alpha i}\right] } & =-\frac{i}{2}\left(\mathcal{Q}_{i} \Gamma_{\hat{\mu} \hat{\nu}}\right)_{\alpha}, \\
{\left[\mathcal{B}_{5}, \mathcal{Q}_{\alpha i}\right] } & =i \epsilon_{i j} \mathcal{Q}_{\alpha j} .
\end{aligned}
$$

Here $\mathcal{M}_{\hat{\mu} \hat{\nu}}$ and $\mathcal{B}_{5}$ are real (Hermitian) while $\mathcal{Q}_{\alpha i}$ are complex and satisfy the symplectic $\mathrm{SU}(2)$ Majorana or quaternionic condition

$$
\mathcal{Q}_{\alpha i}^{\dagger}=-\mathcal{Q}_{\beta j} i \epsilon^{i j}\left(\Gamma_{4}\right)^{\beta}{ }_{\alpha} .
$$

The superalgebra $(3.25)$ is $\mathrm{N}=1, \mathrm{D}=4$ de-Sitter superalgebra $U U_{\alpha}(1,1 \mid 1 ; H)$ discussed in [18] 19] and Appendix B. We can conclude that the $k$-deformed Maxwell superalgebra for $k<0$ is isomorphic to $U U_{\alpha}(1,1 \mid 1 ; H) \oplus O(3,1) \oplus U(1)$ with the generators related by

$$
\begin{aligned}
\mathcal{M}_{\mu 4} & =R P_{\mu}, \quad \mathcal{M}_{\mu \nu}=R^{2} Z_{\mu \nu}, \quad \mathcal{B}_{5}=B_{5}+R^{2} B \\
\mathcal{Q}_{\alpha 1} & =\frac{\sqrt{R}}{2} \mathbf{Q}_{\alpha}+i \sqrt{R^{3}} \boldsymbol{\Sigma}_{\alpha}, \quad \mathcal{Q}_{\alpha 2}=\left(\frac{i \sqrt{R}}{2} \mathbf{Q}_{\beta}+\sqrt{R^{3}} \boldsymbol{\Sigma}_{\beta}\right) \Gamma_{4 \alpha}^{\beta}, \\
\mathcal{J}_{\mu \nu} & =M_{\mu \nu}-R^{2} Z_{\mu \nu}, \quad \mathcal{B}=-R^{2} B .
\end{aligned}
$$

We add that the contractions of the deformed algebras $O S p(2 \mid 4) \oplus O(3,1) \oplus U(1)$ and $U U_{\alpha}(1,1 \mid 1 ; H) \oplus O(3,1) \oplus U(1)$ are not unique but other contractions are possible to produce results different from the Maxwell superalgebra (2.1). For example, one could get $\mathrm{N}=2$ Poincarè superalgebra $\oplus O(3,1) \oplus U(1)^{2}$. 


\section{$3.2 s$-deformation}

From the MC equation (3.7) it follows that only the action of the $B_{5}$ generator is changed in comparison with the undeformed algebra (2.1). Denoting the $s$-deformed generator by $B_{5}^{(s)}$, one gets

$$
\begin{aligned}
& {\left[B_{5}^{(s)}, P_{\mu}\right]=i s P_{\mu}, \quad\left[B_{5}^{(s)}, Z_{\mu \nu}\right]=i 2 s Z_{\mu \nu}, \quad\left[B_{5}^{(s)}, B\right]=i 2 s B, \quad\left[B_{5}^{(s)}, M_{\rho \sigma}\right]=0,} \\
& {\left[B_{5}^{(s)}, \mathbf{Q}_{\alpha}\right]=i\left(\mathbf{Q}\left(\frac{s}{2}-\gamma_{5}\right)\right)_{\alpha}, \quad\left[B_{5}^{(s)}, \boldsymbol{\Sigma}_{\alpha}\right]=i\left(\boldsymbol{\Sigma}\left(\frac{3 s}{2}+\gamma_{5}\right)\right)_{\alpha} .}
\end{aligned}
$$

The generator $B_{5}^{(s)}$ acts on all generators as a dilatation operator and produces as well the chiral rotations for the fermionic generators. Note also that the generator $B$, after the $s$-deformation, ceases to be central. From (2.1), (2.5) and (3.28) it follows that the deformed chiral generator $B_{5}^{(s)}$ can be represented as

$$
B_{5}^{(s)}=B_{5}+s \mathcal{D} .
$$

Because of $\left[\mathcal{D}, B_{5}\right]=0$, the relation (3.29) selects a one-parameter subalgebra from the twodimensional Abelian subalgebra $\left(\mathcal{D}, B_{5}\right)$ of the Maxwell-Weyl superalgebra. We conclude therefore that the $s$-deformed $\mathrm{D}=4$ Maxwell superalgebra is embedded in the Maxwell-Weyl superalgebra considered in section 2 .

\section{4. $\mathrm{D}=3$ Maxwell superalgebra and its deformations}

\section{1 $\mathrm{D}=3$ Maxwell superalgebra}

The $\mathrm{D}=3$ Majorana spinors are two-component, and $O(2,1)$ gamma matrices in Majorana representation can be expressed in terms of the Pauli matrices $\sigma_{i}$ as follows

$$
C=\gamma_{0}=i \sigma_{2}, \quad \gamma_{1}=\sigma_{1}, \quad \gamma_{2}=\sigma_{3} .
$$

Because the chiral product of the three matrices (4.1) is the identity matrix we cannot accommodate the generators $B$ and $B_{5}$ in $\mathrm{D}=3$ Maxwell superalgebra, which by analogy with the formulae (2.1) takes the following form

$$
\begin{aligned}
{\left[P_{\mu}, P_{\nu}\right] } & =i Z_{\mu \nu}, \quad\left[P_{\mu}, \mathbf{Q}_{\alpha}\right]=-i \boldsymbol{\Sigma}_{\beta}\left(\gamma_{\mu}\right)_{\alpha}^{\beta}, \\
\left\{\mathbf{Q}_{\alpha}, \mathbf{Q}_{\beta}\right\} & =2\left(C \gamma^{\mu}\right)_{\alpha \beta} P_{\mu}, \quad\left\{\mathbf{Q}_{\alpha}, \boldsymbol{\Sigma}_{\beta}\right\}=\frac{1}{2}\left(C \gamma^{\mu \nu}\right)_{\alpha \beta} Z_{\mu \nu}, \\
{\left[P_{\mu}, M_{\rho \sigma}\right] } & =-i \eta_{\mu[\rho} P_{\sigma]}, \quad\left[Z_{\mu \nu}, M_{\rho \sigma}\right]=-i \eta_{\nu[\rho} Z_{|\mu| \sigma]}+i \eta_{\mu[\rho} Z_{|\nu| \sigma]}, \\
{\left[M_{\rho \sigma}, \mathbf{Q}_{\alpha}\right] } & =-\frac{i}{2}\left(\mathbf{Q} \gamma_{\rho \sigma}\right)_{\alpha}, \quad\left[M_{\rho \sigma}, \boldsymbol{\Sigma}_{\alpha}\right]=-\frac{i}{2}\left(\mathbf{\Sigma} \gamma_{\rho \sigma}\right)_{\alpha}, \\
{\left[M_{\mu \nu}, M_{\rho \sigma}\right] } & =-i \eta_{\nu[\rho} M_{|\mu| \sigma]}+i \eta_{\mu[\rho} M_{|\nu| \sigma]} .
\end{aligned}
$$

The Jacobi identities of this algebra hold using the identity $\left(C \gamma_{\mu}\right)_{(\alpha \beta}\left(C \gamma^{\mu}\right)_{\gamma \delta)}=0$, for totally symmetric $(\alpha \beta \gamma \delta)$. We would like to mention that the $\mathrm{D}=3$ Maxwell algebra was considered earlier under the name of extended Poincarè algebra [15] 16]. The $\mathrm{D}=3$ Maxwell superalgebra (4.2) is given here for the first time. 


\subsection{Deformations of $\mathrm{D}=3$ Maxwell superalgebra}

In $\mathrm{D}=3$ dimensions there are no $B$ and $B_{5}$ generators and there is no $s$-deformation. On the other hand the presence of the totally antisymmetric tensor $\epsilon^{\mu \nu \rho}$ leads to a new deformation, as in the bosonic case ( $b$-deformation in [8]), in addition to the $k$-deformation (3.3):

$$
\begin{aligned}
d L_{M}^{\mu \nu}+L_{M}^{\mu \rho} \eta_{\rho \sigma} L_{M}^{\sigma \nu} & =0 \\
d L_{P}^{\mu}+L_{M}^{\mu \nu} L_{P \nu}-i \overline{\mathbf{L}} \gamma^{\mu} \mathbf{L} & =c \epsilon_{\rho \sigma}^{\mu} L_{P}^{\rho} L_{P}^{\sigma},+k\left(L_{Z}^{\mu \nu} L_{P \nu}+\frac{i}{4} \overline{\mathbf{L}_{\Sigma}} \gamma^{\mu} \mathbf{L}_{\Sigma}\right) \\
d L_{Z}^{\mu \nu}+L_{M}^{\mu \rho} \eta_{\rho \sigma} L_{Z}^{\sigma \nu}+L_{Z}^{\mu \rho} \eta_{\rho \sigma} L_{M}^{\sigma \nu}-L_{P}^{\mu} L_{P}^{\nu}-i \overline{\mathbf{L}} \gamma^{\mu \nu} \mathbf{L}_{\Sigma}=-\frac{c}{2} i \overline{\mathbf{L}_{\Sigma}} \gamma^{\mu \nu} \mathbf{L}_{\Sigma}+k L_{Z}^{\mu \rho} L_{Z \rho}{ }^{\nu} & \\
d \mathbf{L}^{\alpha}+\frac{1}{4} L_{M}^{\mu \nu}\left(\gamma_{\mu \nu} \mathbf{L}\right)^{\alpha} & =-c L_{P}^{\mu}\left(\gamma_{\mu} \mathbf{L}\right)^{\alpha}+\frac{k}{4}\left(L_{Z}^{\mu \nu}\left(\gamma_{\mu \nu} \mathbf{L}\right)^{\alpha}-L_{P}^{\mu}\left(\gamma_{\mu} \mathbf{L}_{\Sigma}\right)^{\alpha}\right) \\
d \mathbf{L}_{\Sigma}^{\alpha}+\frac{1}{4} L_{M}^{\mu \nu}\left(\gamma_{\mu \nu} \mathbf{L}_{\Sigma}\right)^{\alpha}+L_{P}^{\mu}\left(\gamma_{\mu} \mathbf{L}\right)^{\alpha} & =\frac{k}{4} L_{Z}^{\mu \nu}\left(\gamma_{\mu \nu} \mathbf{L}_{\Sigma}\right)^{\alpha}
\end{aligned}
$$

where $c, k$ are the deformation parameters having the mass dimension $[k]=2,[c]=1$. $\mathbf{L}$ and $\mathbf{L}_{\Sigma}$ are 2-component Majorana spinors. We will examine the symmetry structure of these deformations in the three cases, (1) $k^{+}$-deformation for $k>0,(2) k^{-}$-deformation for $k<0$ and (3) $c$-deformation.

\subsection{1 $k^{+}$-deformation}

In the case $k>0, c=0$ the $\mathrm{MC}$ equation is written as

$$
\begin{aligned}
d L_{\mathcal{J}}^{\mu}+\frac{1}{2} \epsilon^{\mu}{ }_{\nu \rho} L_{\mathcal{J}}^{\nu} L_{\mathcal{J}}^{\rho} & =0 \\
d L_{ \pm}^{\mu}+\frac{1}{2} \epsilon^{\mu}{ }_{\nu \rho} L_{ \pm}^{\nu} L_{ \pm}^{\rho} \mp i \mathbf{L}_{ \pm} C \gamma^{\mu} \mathbf{L}_{ \pm} & =0, \quad d \mathbf{L}_{ \pm}^{\alpha}-\frac{1}{2} L_{ \pm}^{\mu}\left(\gamma_{\mu} \mathbf{L}_{ \pm}\right)^{\alpha}=0
\end{aligned}
$$

where

$$
L_{\mathcal{J}}^{\mu}=\frac{1}{2} \epsilon^{\mu}{ }_{\nu \rho} L_{M}^{\nu \rho}, \quad L_{ \pm}^{\mu}=\frac{1}{2} \epsilon^{\mu}{ }_{\nu \rho}\left(L_{M}^{\nu \rho}-\frac{L_{Z}^{\nu \rho}}{R^{2}}\right) \pm \frac{L_{P}^{\mu}}{R}, \quad \mathbf{L}_{ \pm}^{\alpha}=\frac{\mathbf{L}^{\alpha}}{R^{1 / 2}} \mp \frac{\mathbf{L}_{\Sigma}^{\alpha}}{2 R^{3 / 2}}
$$

and $\mathbf{L}_{ \pm}$are two independent Majorana spinors.

The dual algebra for the generators, defined by using the $\mathrm{MC}$ one form

$$
\Omega=\mathcal{J}_{\mu} L_{\mathcal{J}}^{\mu}+\mathcal{M}_{\mu}^{+} L_{+}^{\mu}+\mathcal{M}_{\mu}^{-} L_{-}^{\mu}+\mathcal{Q}_{\alpha}^{+} \mathbf{L}_{+}^{\alpha}+\mathcal{Q}_{\alpha}^{-} \mathbf{L}_{-}^{\alpha}
$$

becomes

$$
\begin{aligned}
{\left[\mathcal{J}_{\mu}, \mathcal{J}_{\nu}\right] } & =-i \epsilon_{\mu \nu \rho} \mathcal{J}^{\rho}, \\
{\left[\mathcal{M}_{\mu}^{ \pm}, \mathcal{M}_{\nu}^{ \pm}\right] } & =-i \epsilon_{\mu \nu \rho} \mathcal{M}^{ \pm \rho}, \quad\left[\mathcal{M}_{\mu}^{ \pm}, \mathcal{Q}_{\alpha}^{ \pm}\right]=\frac{i}{2}\left(\mathcal{Q}^{ \pm} \gamma_{\mu}\right)_{\alpha} \\
\left\{\mathcal{Q}_{\alpha}^{ \pm}, \mathcal{Q}_{\beta}^{ \pm}\right\} & = \pm 2\left(C \gamma^{\mu}\right)_{\alpha \beta} \mathcal{M}_{\mu}^{ \pm} .
\end{aligned}
$$

Here, due to the relation $\sum_{\alpha} \mathcal{Q}_{\alpha}^{ \pm} \mathcal{Q}_{\alpha}^{ \pm}=-\sum_{\alpha}\left|\mathcal{Q}_{\alpha}^{ \pm}\right|^{2}= \pm 2 \mathcal{M}_{0}^{ \pm}$, we obtain the constraints $\mathcal{M}_{0}^{+} \leq 0, \mathcal{M}_{0}^{-} \geq 0$. The $\mathcal{J}_{\mu}$ is the $O(2,1)$ generator and $\left(\mathcal{M}_{\mu}^{ \pm}, \mathcal{Q}_{\alpha}^{ \pm}\right)$generates a pair of real superalgebras $O S p^{ \pm}(1 \mid 2)$. Then the $k^{+}$-deformed super algebra is a direct sum:

$$
k^{+} \text {-deformed superalgebra }=O(2,1) \oplus O S p^{+}(1 \mid 2) \oplus O S p^{-}(1 \mid 2) \text {. }
$$




\subsection{2 $k^{-}$-deformation}

In the case $k<0, c=0$ the MC equation is written as

$$
\begin{aligned}
d L_{\mathcal{J}}^{\mu}+\frac{1}{2} \epsilon^{\mu}{ }_{\nu \rho} L_{\mathcal{J}}^{\nu} L_{\mathcal{J}}^{\rho} & =0 \\
d L_{ \pm}^{\mu}+\frac{1}{2} \epsilon^{\mu}{ }_{\nu \rho} L_{ \pm}^{\nu} L_{ \pm}^{\rho} \pm \mathbf{L}_{ \pm} C \gamma^{\mu} \mathbf{L}_{ \pm} & =0, \quad d \mathbf{L}_{ \pm}^{\alpha}-\frac{1}{2} L_{ \pm}^{\mu}\left(\gamma_{\mu} \mathbf{L}_{ \pm}\right)^{\alpha}=0
\end{aligned}
$$

where

$$
L_{\mathcal{J}}^{\mu}=\frac{1}{2} \epsilon^{\mu}{ }_{\nu \rho} L_{M}^{\nu \rho}, \quad L_{ \pm}^{\mu}=\frac{1}{2} \epsilon^{\mu}{ }_{\nu \rho}\left(L_{M}^{\nu \rho}+\frac{L_{Z}^{\nu \rho}}{R^{2}}\right) \pm i \frac{L_{P}^{\mu}}{R}, \quad \mathbf{L}_{ \pm}^{\alpha}=\frac{\mathbf{L}^{\alpha}}{R^{1 / 2}} \mp i \frac{\mathbf{L}_{\Sigma}^{\alpha}}{2 R^{3 / 2}},
$$

and $L_{+}^{\mu}=L_{-}^{\mu \dagger}, \mathbf{L}_{+}^{\alpha}=\mathbf{L}_{-}^{\alpha \dagger}$. The dual algebra for the generators, defined by using the MC one form

$$
\Omega=\mathcal{J}_{\mu} L_{\mathcal{J}}^{\mu}+\mathcal{M}_{\mu}^{+} L_{+}^{\mu}+\mathcal{M}_{\mu}^{-} L_{-}^{\mu}+\mathcal{Q}_{\alpha}^{+} \mathbf{L}_{+}^{\alpha}+\mathcal{Q}_{\alpha}^{-} \mathbf{L}_{-}^{\alpha}
$$

becomes

$$
\begin{aligned}
{\left[\mathcal{J}_{\mu}, \mathcal{J}_{\nu}\right] } & =-i \epsilon_{\mu \nu \rho} \mathcal{J}^{\rho} \\
{\left[\mathcal{M}_{\mu}^{ \pm}, \mathcal{M}_{\nu}^{ \pm}\right] } & =-i \epsilon_{\mu \nu \rho} \mathcal{M}^{ \pm \rho}, \quad\left[\mathcal{M}_{\mu}^{ \pm}, \mathcal{Q}_{\alpha}^{ \pm}\right]=\frac{i}{2}\left(\mathcal{Q}^{ \pm} \gamma_{\mu}\right)_{\alpha} \\
\left\{\mathcal{Q}_{\alpha}^{ \pm}, \mathcal{Q}_{\beta}^{ \pm}\right\} & = \pm 2 i\left(C \gamma^{\mu}\right)_{\alpha \beta} \mathcal{M}_{\mu}^{ \pm}
\end{aligned}
$$

Note there is a factor $i$ in the $\{\mathcal{Q}, \mathcal{Q}\}$ anticommutator because of the hermiticity requirement

$$
\left(\mathcal{M}_{\mu}^{-}\right)^{\dagger}=\mathcal{M}_{\mu}^{+}, \quad\left(\mathcal{Q}_{\alpha}^{-}\right)^{\dagger}=-\mathcal{Q}_{\alpha}^{+}
$$

Because $\mathcal{Q}_{\alpha}^{+}$and $\mathcal{Q}_{\beta}^{-}$are anticommuting

$$
\left\{\mathcal{Q}_{\alpha}^{+}, \mathcal{Q}_{\beta}^{-}\right\}=-\left\{\mathcal{Q}_{\alpha}^{+}, \mathcal{Q}_{\beta}^{+\dagger}\right\}=0
$$

one obtains that $\sum_{\alpha}\left|\mathcal{Q}_{\alpha}^{ \pm}\right|^{2}=0$ and representations at quantum level of the superalgebra (4.12) lead necessarily to indefinite metric and ghost states 19] 18]. The algebra is a direct sum of $\mathrm{O}(2,1)$ with generator $\mathcal{J}, O S p(1 \mid 2 ; C)$ with complex generators $\mathcal{M}_{+}, \mathcal{Q}_{+}$and $\overline{O S p(1 \mid 2 ; C)}$ with their conjugate generators $\mathcal{M}_{-}, \mathcal{Q}_{-}$. We obtain

$$
k^{-} \text {-deformed superalgebra }=O(2,1) \oplus O S p(1 \mid 2 ; C) \oplus \overline{O S p(1 \mid 2 ; C)} .
$$

\subsection{3 c-deformation}

The $c$-deformation is the case $k=0$ given by

$$
\begin{array}{rlrl}
d L_{\mathcal{J}}^{\mu}+\frac{1}{2} \epsilon^{\mu}{ }_{\nu \rho} L_{\mathcal{J}}^{\nu} L_{\mathcal{J}}^{\rho} & =-2 c i \overline{\tilde{\mathbf{L}}} \gamma^{\mu} \tilde{\mathbf{L}}, \quad & d \tilde{\mathbf{L}}^{\alpha}-\frac{1}{2} L_{\mathcal{J}}^{\mu}\left(\gamma_{\mu} \tilde{\mathbf{L}}\right)^{\alpha}=0, \\
d L_{\mathcal{M}}^{\mu}+\frac{1}{2} \epsilon^{\mu}{ }_{\nu \rho} L_{\mathcal{M}}^{\nu} L_{\mathcal{M}}^{\rho} & =0, \\
d L_{\mathcal{P}}^{\mu}+\epsilon^{\mu}{ }_{\nu \rho} L_{\mathcal{M}}^{\nu} L_{\mathcal{P}}^{\rho} & =-\frac{i}{2} \overline{\tilde{\mathbf{L}}_{\Sigma}} \gamma^{\mu} \tilde{\mathbf{L}}_{\Sigma}, & d \tilde{\mathbf{L}}_{\Sigma}^{\alpha}-\frac{1}{2} L_{\mathcal{M}}^{\mu}\left(\gamma_{\mu} \tilde{\mathbf{L}}_{\Sigma}\right)^{\alpha}=0 .
\end{array}
$$


where

$$
L_{\mathcal{J}}^{\mu}=-2 c L_{P}^{\mu}+\frac{1}{2} \epsilon^{\mu}{ }_{\nu \rho} L_{M}^{\nu \rho}, \quad L_{\mathcal{P}}^{\mu}=c \frac{1}{2} \epsilon^{\mu}{ }_{\nu \rho} L_{Z}^{\nu \rho}-\frac{1}{2} L_{P}^{\mu}, \quad L_{\mathcal{M}}^{\mu}=\frac{1}{2} \epsilon^{\mu}{ }_{\nu \rho} L_{M}^{\nu \rho},
$$

and

$$
\tilde{\mathbf{L}}^{\alpha}=\mathbf{L}^{\alpha}, \quad \tilde{\mathbf{L}}_{\Sigma}^{\alpha}=\mathbf{L}^{\alpha}-c \mathbf{L}_{\Sigma}^{\alpha}, \quad[\tilde{\mathbf{L}}]=\left[\tilde{\mathbf{L}}_{\Sigma}\right]=-1 / 2
$$

The dual algebra for the generators, defined by using the $\mathrm{MC}$ one form

$$
\Omega=\mathcal{J}_{\mu} L_{\mathcal{J}}^{\mu}+\tilde{\mathbf{Q}}_{\alpha} \tilde{\mathbf{L}}^{\alpha}+\mathcal{M}_{\mu} L_{\mathcal{M}}^{\mu}+\mathcal{P}_{\mu} L_{\mathcal{P}}^{\mu}+\tilde{\boldsymbol{\Sigma}}_{\alpha} \tilde{\mathbf{L}}_{\Sigma}^{\alpha}
$$

is

$$
\begin{aligned}
{\left[\mathcal{J}_{\mu}, \mathcal{J}_{\nu}\right]=-i \epsilon_{\mu \nu \rho} \mathcal{J}^{\rho}, } & \left\{\tilde{\mathbf{Q}}_{\alpha}, \tilde{\mathbf{Q}}_{\beta}\right\}=-4 c\left(C \gamma^{\mu}\right)_{\alpha \beta} \mathcal{J}_{\mu} \\
{\left[\mathcal{M}_{\mu}, \mathcal{M}_{\nu}\right]=-i \epsilon_{\mu \nu \rho} \mathcal{M}^{\rho}, } & {\left[\mathcal{M}_{\mu}, \tilde{\boldsymbol{\Sigma}}_{\alpha}\right]=\frac{i}{2}\left(\tilde{\boldsymbol{\Sigma}} \gamma_{\mu}\right)_{\alpha} } \\
{\left[\mathcal{P}_{\mu}, \mathcal{M}_{\nu}\right]=-i \epsilon_{\mu \nu \rho} \mathcal{P}^{\rho}, } & \left\{\tilde{\boldsymbol{\Sigma}}_{\alpha}, \tilde{\boldsymbol{\Sigma}}_{\beta}\right\}=-\left(C \gamma^{\mu}\right)_{\alpha \beta} \mathcal{P}_{\mu} .
\end{aligned}
$$

Here $\tilde{\mathbf{Q}}_{\alpha}$ and $\tilde{\boldsymbol{\Sigma}}_{\alpha}$ are independent Majorana spinors. The algebra with generators $(\mathcal{J}, \tilde{Q})$ is $\operatorname{OSp}(1 \mid 2)$ and one with $(\mathcal{M}, \mathcal{P}, \tilde{\Sigma})$ is $\mathrm{N}=1, \mathrm{D}=3$ Poincarè superalgebra, i.e.,

$$
c-\text { deformed superalgebra }=O S p(1 \mid 2) \oplus(\mathrm{N}=1 \mathrm{D}=3 \text { superPoincare }) .
$$

The $c$-deformation is the supersymmetrization of the degenerate case of the two parameter deformation of $\mathrm{D}=3$ bosonic Maxwell algebra [8].

\section{Casimir Operators of the Maxwell superalgebras}

In this section we discuss how the Casimir operators of Maxwell superalgebras can be obtained as contractions of their deformed counterparts and their subalgebras.

\subsection{Casimir Operators of the Maxwell superalgebras in $\mathrm{D}=4$}

In four dimensions there are four Casimir operators in the bosonic Maxwell algebra [2] [5],

$$
\begin{aligned}
& C_{1}=\frac{1}{2} Z_{\mu \nu}^{2}, \quad C_{2}=\frac{1}{2}\left(Z_{\mu \nu} \tilde{Z}^{\mu \nu}\right), \\
& C_{3}=P_{\mu}^{2}+M_{\mu \nu} Z^{\mu \nu}, \quad C_{4}=\left(P^{\nu} \tilde{Z}_{\nu \mu}\right)^{2}+\frac{1}{4}\left(Z_{\mu \nu} \tilde{Z}^{\mu \nu}\right)\left(M_{\rho \sigma} \tilde{Z}^{\rho \sigma}\right),
\end{aligned}
$$

where $\tilde{Z}^{\mu \nu}=\frac{1}{2} \epsilon^{\mu \nu \rho \sigma} Z_{\rho \sigma}$. As shown in the subsection 3.1.1 the $k$-deformed Maxwell superalgebra for $k>0$ is isomorphic to $O(3,1) \oplus O S p(2 \mid 4) \oplus U(1)$; thus there are $6=2+3+1$ Casimir operators. In the contracted algebra four of the Casimir operators are the supersymmetrized counterparts of (5.1) given in [10]. In addition there is one $U(1)$ Casimir $\mathcal{B}$ and one related to the 6th order $O S p(2 \mid 4)$ Casimir operator. 


\subsubsection{Casimir operators of $\mathrm{O}(\mathrm{N})$ algebras}

For $O(N)$ (with indefinite metric $\eta= \pm 1$ ) with generators $M_{A B}=\eta_{A C} M^{C}{ }_{B}=-M_{B A}$, the $n$-th order traces

$$
C^{(n)}=\frac{1}{2} \operatorname{tr}\left(M^{n}\right)=\frac{1}{2} M_{A_{2}}^{A_{1}} M^{A_{2}}{ }_{A_{3}} \ldots M^{A_{n}} A_{1}
$$

commute with every generator and are therefore Casimir operators for even $n$. (For odd $n, C^{(n)}=0$ identically.) There are $\left[\frac{N}{2}\right]$ independent Casimir operators since the $C^{(n)}$ for $n>N$ are expressed as polynomial functions of lower ones using the Cayley-Hamilton theorem.

For $O(3,1)$ there are two independent Casimir operators,

$$
\begin{aligned}
C_{J}^{(2)} & =\frac{1}{2} \mathcal{J}^{\mu}{ }_{\nu} \mathcal{J}^{\nu}{ }_{\mu}=-\frac{1}{2} \mathcal{J}_{\mu \nu} \mathcal{J}^{\mu \nu}, \\
C_{J}^{(4)} & =\frac{1}{2} \operatorname{tr}\left(\mathcal{J}^{4}\right)=\left(C_{J}^{(2)}\right)^{2}-2\left(\epsilon^{\mu \nu \rho \sigma} \mathcal{J}_{\mu \nu} \mathcal{J}_{\rho \sigma}\right)^{2} .
\end{aligned}
$$

The independent Casimir operators of $O(3,1)$ are thus

$$
C_{J}^{1}=\frac{1}{2} \mathcal{J}_{\mu \nu} \mathcal{J}^{\mu \nu}=\frac{1}{2}(\mathcal{J} \mathcal{J}), \quad C_{J}^{2}=\frac{1}{4} \epsilon^{\mu \nu \rho \sigma} \mathcal{J}_{\mu \nu} \mathcal{J}_{\rho \sigma}=\frac{1}{2}(\mathcal{J} \tilde{\mathcal{J}})
$$

\subsection{2 $O S p(2 \mid 4) \quad$ Casimir operators}

There are $\left[\frac{n}{2}\right]+\left[\frac{m}{2}\right]$ Casimir operators in the superalgebra $O S p(m \mid n)$ [27]. The generators of $O S p(2 \mid 4)$ are expressed as a graded antisymmetric $O S p(2 \mid 4)$ supermatrix $M_{A B}$ with the graded symmetric $O S p(2 \mid 4)$ metric $\eta^{A B},(A=(i, \alpha) ; i=1,2, \alpha=1,2,3,4)$,

$$
\begin{array}{ccc}
M_{A B} & =\left(\begin{array}{ccc}
0 & \mathcal{B}_{5} & \mathcal{Q}_{1 \beta} \\
-\mathcal{B}_{5} & 0 & \mathcal{Q}_{2 \beta} \\
\mathcal{Q}_{1 \alpha} & \mathcal{Q}_{2 \alpha} & M_{\alpha \beta}
\end{array}\right), & \eta^{A B}=\left(\begin{array}{ccc}
1 & 0 & 0 \\
0 & 1 & 0 \\
0 & 0 & -i\left(C^{-1}\right)^{\alpha \beta}
\end{array}\right), \\
M^{A}{ }_{B}=\eta^{A C} M_{C B}=\left(\begin{array}{ccc}
0 & \mathcal{B}_{5} & \mathcal{Q}_{1 \beta} \\
-\mathcal{B}_{5} & 0 & \mathcal{Q}_{2 \beta} \\
-i C^{-1 \alpha \gamma} \mathcal{Q}_{1 \gamma} & -i C^{-1 \alpha \gamma} \mathcal{Q}_{2 \gamma} & -i C^{-1 \alpha \gamma} M_{\gamma \beta}
\end{array}\right) .
\end{array}
$$

The 10 symmetric $\operatorname{Sp}(4)$ generators $M_{\alpha \beta}$ are expressed in terms of the 10 antisymmetric $\mathrm{O}(3,2)$ generators $\mathcal{M}_{\hat{\mu} \hat{\nu}}$ as

$$
M_{\alpha \beta}=-\frac{i}{2}\left(C \Gamma^{\hat{\mu} \hat{\nu}}\right)_{\alpha \beta} \mathcal{M}_{\hat{\mu} \hat{\nu}}
$$

The $O S p(2 \mid 4)$ algebra is expressed as

$$
\begin{aligned}
{\left[M_{A B}, M_{C D}\right]_{ \pm}=} & (-i)\left(M_{A D} \eta_{C B}(-)^{C}+(-)^{(A+1)(B+1)+C} M_{B D} \eta_{C A}\right. \\
& \left.+M_{A C} \eta_{D B}(-)^{C D+C+1}+(-)^{(A B+A+B+C D+C)} M_{B C} \eta_{D A}\right),
\end{aligned}
$$

where $(-)^{A}=+1$ for even $(\mathrm{O}(2))$ indices $A$ and $(-)^{A}=-1$ for odd $(\operatorname{Sp}(4))$ ones. 
As in the $\mathrm{O}(\mathrm{N})$ case we construct the Casimir operators of $O S p(2 \mid 4)$ using its supertraces

$$
C^{(n)}=-\frac{1}{2} \operatorname{str}\left(M^{n}\right)=-\frac{1}{2}(-1)^{A_{1}} M_{A_{2}}^{A_{1}} M_{A_{3}}^{A_{2}} \ldots M_{A_{1}}^{A_{n}} .
$$

For odd $n$ they vanish identically due to graded antisymmetry of $M$. There are thus three independent Casimir operators $C^{(2)}, C^{(4)}, C^{(6)}$. Higher power Casimir operators are not independent but rational functions of these three as follows from the super CayleyHamilton theorem [28].

The explicit forms of these Casimir operators are

$$
\begin{aligned}
C^{(2)} & =-\frac{1}{2} \operatorname{str}\left(M^{2}\right)=\mathcal{B}_{5}^{2}-\left(\mathcal{M}^{2}\right)+\left(i \mathcal{Q}_{i} C^{-1} \mathcal{Q}_{i}\right) \\
C^{(4)} & =-\frac{1}{2} \operatorname{str}\left(M^{4}\right)=-\left(C^{(2)}\right)^{2}+\tilde{C}_{4} \\
\tilde{C}_{4} & =-2 \mathcal{B}_{5}^{2}\left(\mathcal{M}^{2}\right)+\frac{3}{2}\left(\mathcal{M}^{2}\right)^{2}+2(\mathcal{M} \tilde{\mathcal{M}})^{2} \\
& +\mathcal{B}_{5} \mathcal{M}_{\hat{\mu} \hat{\nu}}\left(\epsilon^{i j} i \mathcal{Q}_{i} \Gamma^{\hat{\mu} \hat{\nu}} C^{-1} \mathcal{Q}_{j}\right)-\frac{1}{8}\left(\epsilon^{i j} i \mathcal{Q}_{i} \Gamma^{\hat{\mu} \hat{\nu}} C^{-1} \mathcal{Q}_{j}\right)^{2} \\
& -3\left(\mathcal{M}^{2}\right)\left(i \mathcal{Q}_{i} C^{-1} \mathcal{Q}_{i}\right)+2(\mathcal{M} \tilde{\mathcal{M}})^{\hat{\mu}}\left(i \mathcal{Q}_{i} \Gamma_{\hat{\mu}} C^{-1} \mathcal{Q}_{i}\right) \\
C^{(6)} & =-\frac{1}{2} \operatorname{str}\left(M^{6}\right)=-\frac{1}{2}\left(C^{(2)}\right)^{3}-\frac{3}{2}\left(C^{(2)}\right)\left(C^{(4)}\right)+\tilde{C}_{6} \\
\tilde{C}_{6} & =\mathcal{B}_{5}^{2}\left(\frac{9}{4}\left(\mathcal{M}^{2}\right)^{2}+3\left(\mathcal{M} \tilde{\mathcal{M}}^{2}\right)-\frac{3}{2}\left(\mathcal{M}^{2}\right)^{3}-6\left(\mathcal{M}^{2}\right)\left(\mathcal{M} \mathcal{M}^{2}\right.\right. \\
& +\mathcal{B}_{5}\left(-3\left(\mathcal{M}^{3}\right)^{\hat{\mu} \hat{\nu}}-\frac{15}{4}\left(\mathcal{M}^{2}\right) \mathcal{M}^{\hat{\mu} \hat{\nu}}\right)\left(\epsilon^{i j} i \mathcal{Q}_{i} \Gamma_{\hat{\mu} \hat{\nu}} C^{-1} \mathcal{Q}_{j}\right) \\
& -6\left(\mathcal{M}^{2}\right)(\mathcal{M} \tilde{\mathcal{M}})^{\hat{\mu}}\left(i \mathcal{Q}_{i} \Gamma_{\hat{\mu}} C^{-1} \mathcal{Q}_{i}\right)+\left(\frac{9}{2}\left(\mathcal{M}^{2}\right)^{2}+6(\mathcal{M} \tilde{\mathcal{M}})^{2}\right)\left(i \mathcal{Q}_{i} C^{-1} \mathcal{Q}_{i}\right) \\
& +\frac{1}{4}\left(\mathcal{M}^{2}\right)^{\hat{\mu} \hat{\nu}}\left(\epsilon^{i j} i \mathcal{Q}_{i} \Gamma_{\hat{\mu} \hat{\rho}} C^{-1} \mathcal{Q}_{j}\right) \eta^{\hat{\rho} \hat{\sigma}}\left(\epsilon^{i j} i \mathcal{Q}_{i} \Gamma_{\hat{\sigma} \hat{\nu}} C^{-1} \mathcal{Q}_{j}\right) \\
& +\frac{21}{4}(\mathcal{M} \tilde{\mathcal{M}})^{\hat{\mu}}\left(i \mathcal{Q}_{i} \Gamma_{\hat{\mu}} C^{-1} \mathcal{Q}_{i}\right)\left(i \mathcal{Q}_{i} C^{-1} \mathcal{Q}_{i}\right) \\
& -\frac{11}{16}\left(\mathcal{M}^{2}\right)\left(5\left(i \mathcal{Q}_{i} C^{-1} \mathcal{Q}_{i}\right)^{2}+\left(i \mathcal{Q}_{i} \Gamma_{\hat{\mu}} C^{-1} \mathcal{Q}_{i}\right)^{2}\right)
\end{aligned}
$$

where $\tilde{C}_{4}, \tilde{C}_{6}$ are defined by subtracting terms with higher power of $\mathcal{B}_{5}$ and

$$
\begin{aligned}
\left(\mathcal{M}^{2}\right) & =\left(\mathcal{M}^{\hat{\mu} \hat{\nu}} \mathcal{M}_{\hat{\mu} \hat{\nu}}\right), \quad(\mathcal{M} \tilde{\mathcal{M}})^{\hat{\mu}}=\frac{1}{4} \epsilon^{\hat{\mu} \hat{\nu} \hat{\rho} \hat{\sigma} \hat{\lambda}} \mathcal{M}_{\hat{\nu} \hat{\rho}} \mathcal{M}_{\hat{\sigma} \hat{\lambda}}, \\
\left(\mathcal{M}^{2}\right)^{\hat{\mu} \hat{\nu}} & =\mathcal{M}^{\hat{\mu} \hat{\rho}} \mathcal{M}_{\hat{\rho}}^{\hat{\nu}}, \quad\left(\mathcal{M}^{3}\right)^{\hat{\mu} \hat{\nu}}=\mathcal{M}^{\hat{\mu} \hat{\rho}} \mathcal{M}_{\hat{\rho} \hat{\sigma}} \mathcal{M}^{\hat{\sigma} \hat{\nu}} .
\end{aligned}
$$

These expressions are not unique due to the identity

$$
10\left(i \mathcal{Q}_{i} C^{-1} \mathcal{Q}_{i}\right)^{2}+2\left(i \mathcal{Q}_{i} \Gamma_{\hat{\mu}} C^{-1} \mathcal{Q}_{i}\right)^{2}+\left(\epsilon^{i j} i \mathcal{Q}_{i} \Gamma_{\hat{\mu} \hat{\nu}} C^{-1} \mathcal{Q}_{j}\right)^{2}=0
$$

which is proved using completeness of the $\mathrm{O}(3,2)$ gamma matrices.

\subsubsection{Casimir operators of the Maxwell superalgebra by contraction}

We have shown that the $k$-deformed Maxwell superalgebra for $k>0$ is $O(3,1) \oplus O S p(2 \mid 4) \oplus$ $U(1)_{\mathcal{B}}$ and the Maxwell superalgebra is obtained by the contraction $k \rightarrow 0(R \rightarrow \infty)$. We 
have obtained six Casimir operators of the $k$-deformed Maxwell superalgebra: two of $O(3,1)$ in (5.4), three of $O S p(2 \mid 4)$ in (5.10)-(5.12) and $\mathcal{B}$ of $U(1)$. The Casimir operators of the Maxwell superalgebra can be found by contraction of those in the $k$-deformed algebra.

Using the relations of generators $(3.19)$ we first rewrite the Casimir operators of $U(1)_{\mathcal{B}}$ and $O(3,1)$ as

$$
\begin{aligned}
& C_{B}=\mathcal{B}=\frac{1}{k} B \\
& C_{J}^{1}=\frac{1}{2}(\mathcal{J} \mathcal{J})=\frac{1}{2}\left(M_{\mu \nu}+\frac{Z_{\mu \nu}}{k}\right)\left(M^{\mu \nu}+\frac{Z^{\mu \nu}}{k}\right), \\
& C_{J}^{2}=\frac{1}{4} \epsilon^{\mu \nu \rho \sigma} \mathcal{J}_{\mu \nu} \mathcal{J}_{\rho \sigma}=\frac{1}{4} \epsilon^{\mu \nu \rho \sigma}\left(M_{\mu \nu}+\frac{Z_{\mu \nu}}{k}\right)\left(M_{\rho \sigma}+\frac{Z_{\rho \sigma}}{k}\right) .
\end{aligned}
$$

In the contraction the leading terms give the Casimir operators of the contracted algebra. In this way we get three Casimir operators of Maxwell superalgebra as

$$
\begin{aligned}
\mathcal{C}_{B} & =\lim _{k \rightarrow 0} k C_{B}=B, \\
\mathcal{C}_{J}^{1} & =\lim _{k \rightarrow 0} k^{2} C_{J}^{1}=\frac{1}{2}(Z Z), \\
\mathcal{C}_{J}^{2} & =\lim _{k \rightarrow 0} k^{2} C_{J}^{2}=\frac{1}{2}(Z \tilde{Z}) .
\end{aligned}
$$

For the $O S p(2 \mid 4)$ Casimir operator

$$
C^{(2)}=\mathcal{B}_{5}^{2}-(\mathcal{M M})+\left(i \mathcal{Q}_{i} C^{-1} \mathcal{Q}_{i}\right)=\frac{1}{k^{2}}\left(B^{2}-(Z Z)\right)+\frac{1}{k}\left(\mathcal{C}_{2}\right)+\ldots
$$

The leading order term $\left(\mathcal{C}_{B}\right)^{2}-2\left(\mathcal{C}_{J}^{1}\right)$ is a function of the Casimir operators, which we have found above. In this case the independent Casimir appears as the next leading term [29]. If we consider the combination in which the leading $k^{-2}$ terms cancel

$$
\mathcal{C}_{2}=\lim _{k \rightarrow 0} k\left(C_{2}+2\left(C_{J}^{1}\right)-\left(C_{B}\right)^{2}\right)=2\left(P^{2}+(M Z)+i \boldsymbol{\Sigma} C^{-1} \mathbf{Q}-B_{5} B\right)
$$

we obtain the mass Casimir of the Maxwell superalgebra found in [10]. Note that subleading term $(M Z)$ in $\left(C_{J}^{1}\right)$ also contributes to the $\mathcal{C}_{2}$ term.

In the same way the leading $k^{-4}$ terms in $\tilde{C}_{4}$ are cancelled by a combination of other Casimir operators

$$
\tilde{C}_{4}-6\left(C_{J}^{1}\right)^{2}+2\left(C_{J}^{2}\right)^{2}+4\left(C_{B}\right)^{2}\left(C_{J}^{1}\right)=\frac{1}{k^{3}}\left(\mathcal{C}_{4}\right)+\frac{1}{k^{2}}(\ldots)+
$$

leaving

$$
\begin{aligned}
\mathcal{C}_{4} & =\lim _{k \rightarrow 0} k^{3}\left(\tilde{C}_{4}-6\left(C_{J}^{1}\right)^{2}+2\left(C_{J}^{2}\right)^{2}+4\left(C_{B}\right)^{2}\left(C_{J}^{1}\right)\right)+4\left(\mathcal{C}_{J}^{1}\right)\left(\mathcal{C}_{2}\right) \\
& =8(P \tilde{Z})^{2}+2(Z \tilde{Z})(M \tilde{Z})+\left(4 B^{2}-2(Z Z)\right)\left(P^{2}+(M Z)\right) \\
& -2(Z Z)\left(i \boldsymbol{\Sigma} C^{-1} \mathbf{Q}\right)-2 B Z^{\mu \nu}\left(i \boldsymbol{\Sigma} \gamma_{\mu \nu} \gamma_{5} C^{-1} \mathbf{Q}\right)-2(Z \tilde{Z})\left(i \boldsymbol{\Sigma} \gamma_{5} C^{-1} \mathbf{Q}\right) \\
& +\left(8(P \tilde{Z})^{\mu}+4 B P^{\mu}\right)\left(i \boldsymbol{\Sigma} \gamma_{\mu} \gamma_{5} C^{-1} \boldsymbol{\Sigma}\right)-4\left(i \boldsymbol{\Sigma} \gamma_{5} C^{-1} \boldsymbol{\Sigma}\right)^{2}
\end{aligned}
$$


where $(P \tilde{Z})^{\mu}=P_{\nu} \tilde{Z}^{\nu \mu}$. Finally, the leading $k^{-6}$ terms in $\tilde{C}_{6}$ are cancelled by the following combination of other Casimir operators

$$
\begin{aligned}
\mathcal{C}_{6}= & \lim _{k \rightarrow 0} k^{5}\left(\tilde{C}_{6}+12\left(C_{J}^{1}\right)^{3}-12\left(C_{J}^{1}\right)\left(C_{J}^{2}\right)^{2}-9\left(C_{B}\right)^{2}\left(C_{J}^{1}\right)^{2}+3\left(C_{B}\right)^{2}\left(C_{J}^{2}\right)^{2}\right) \\
& -\left(9\left(\mathcal{C}_{J}^{1}\right)^{2}-3\left(\mathcal{C}_{J}^{2}\right)^{2}\right)\left(\mathcal{C}_{2}\right) \\
= & \left(\frac{9}{2}(Z Z)^{2}-9 B^{2}(Z Z)-\frac{3}{2}(Z \tilde{Z})^{2}\right)((P P)+(M Z)) \\
+ & 12\left(B^{2}-2(Z Z)\right)\left((P \tilde{Z})^{2}+\frac{1}{4}(M \tilde{Z})(Z \tilde{Z})\right) \\
+ & 6(Z Z)(Z \tilde{Z})\left(i \boldsymbol{\Sigma} \gamma_{5} C^{-1} \mathbf{Q}\right)+\frac{3}{2} B\left(3(Z Z) Z^{\mu \nu}-(Z \tilde{Z}) \tilde{Z}^{\mu \nu}\right)\left(i \boldsymbol{\Sigma} \gamma_{\mu \nu} \gamma_{5} C^{-1} \mathbf{Q}\right) \\
- & 3 B\left(5(Z Z) P^{\mu}+4(P Z Z)^{\mu}\right)\left(i \boldsymbol{\Sigma} \gamma_{\mu} \gamma_{5} C^{-1} \boldsymbol{\Sigma}\right)-24(Z Z)(P \tilde{Z})^{\mu}\left(i \boldsymbol{\Sigma} \gamma_{\mu} \gamma_{5} C^{-1} \boldsymbol{\Sigma}\right) \\
+ & \frac{3}{2}\left(3(Z Z)^{2}-(Z \tilde{Z})^{2}\right)\left(i \boldsymbol{\Sigma} C^{-1} \mathbf{Q}\right)+12(Z Z)\left(i \boldsymbol{\Sigma} \gamma_{5} C^{-1} \boldsymbol{\Sigma}\right)^{2},
\end{aligned}
$$

where $(P Z Z)^{\mu}=P^{\nu} Z_{\nu \rho} Z^{\rho \mu}$. When all fermions vanish, $\mathcal{C}_{6}$ becomes a function of lower order Casimir operators. This explains why $\mathcal{C}_{6}$ does not appear in the bosonic Maxwell algebra.

In summary there are 6 Casimir operators in the Maxwell superalgebra, $\mathcal{C}_{B}, \mathcal{C}_{J}^{1}, \mathcal{C}_{J}^{2}, \mathcal{C}_{2}$, $\mathcal{C}_{4}, \mathcal{C}_{6}$. Note that in the defining equations there appear products of generators, which are not (anti)commuting. A careful investigation shows that we obtain the Hermitian Casimir operators if we use the operator ordering presented above.

\subsection{Casimir operators of $\mathrm{D}=3$ Maxwell superalgebras}

In three dimensions the Maxwell superalgebra is also obtained from the deformed algebras by contraction. Similarly as in four dimensions we obtain the Casimir operators from those of the deformed algebra by contraction. The $k^{+}$-deformed algebra is the direct sum of $O(2,1) \oplus O S p(1 \mid 2) \oplus O S p(1 \mid 2)$ and its Casimir operators are

$$
C_{\mathcal{J}}=\mathcal{J}_{\mu} \mathcal{J}^{\mu}
$$

for the $O(2,1)$ and

$$
C^{ \pm}=4 \mathcal{M}_{\mu}^{ \pm} \mathcal{M}^{ \pm \mu} \mp i \mathbf{Q}^{ \pm} C^{-1} \mathbf{Q}^{ \pm}
$$

from each of the $O S p(1 \mid 2)$ 's. We recall that there is only one Casimir operator in $O S p(1 \mid 2)$.

The Casimir operators of Maxwell superalgebra in $\mathrm{D}=3$ is obtained by contracting them. Using relation of generators dual to 4.5

$$
\begin{aligned}
& C_{\mathcal{J}}=R^{4} Z^{2}+R^{2} 2 M Z+M^{2}, \\
& C^{ \pm}=R^{4} Z^{2} \pm R^{3}\left(2 P Z-i \boldsymbol{\Sigma} C^{-1} \boldsymbol{\Sigma}\right)+R^{2}\left(P^{2}+i \mathbf{Q} C^{-1} \boldsymbol{\Sigma}\right) \mp R\left(\mathbf{Q} C^{-1} \mathbf{Q}\right)
\end{aligned}
$$

we get three Casimir operators of the Maxwell superalgebra in three dimensions,

$$
\begin{aligned}
C_{1} & =\lim _{R \rightarrow \infty} \frac{1}{R^{4}} C_{\mathcal{J}}=Z_{\mu} Z^{\mu}, \\
C_{2} & =\lim _{R \rightarrow \infty} \frac{1}{2 R^{2}}\left(\left(C^{+}+C^{-}\right)-2 C_{\mathcal{J}}\right)=P_{\mu}^{2}-2 M_{\mu} Z^{\mu}+i \mathbf{Q} C^{-1} \boldsymbol{\Sigma}, \\
C_{3} & =\lim _{R \rightarrow \infty} \frac{1}{2 R^{4}}\left(C^{+}-C^{-}\right)=2 P_{\mu} Z^{\mu}-i \boldsymbol{\Sigma} C^{-1} \boldsymbol{\Sigma} .
\end{aligned}
$$




\section{Conclusions and Outlook}

In this paper we presented all deformations of $\mathrm{D}=3$ and $\mathrm{D}=4$ Maxwell superalgebras. The operations of deformation and supersymmetrization of Maxwell algebra can be done in different order resulting in different superalgebras (see Fig.1).

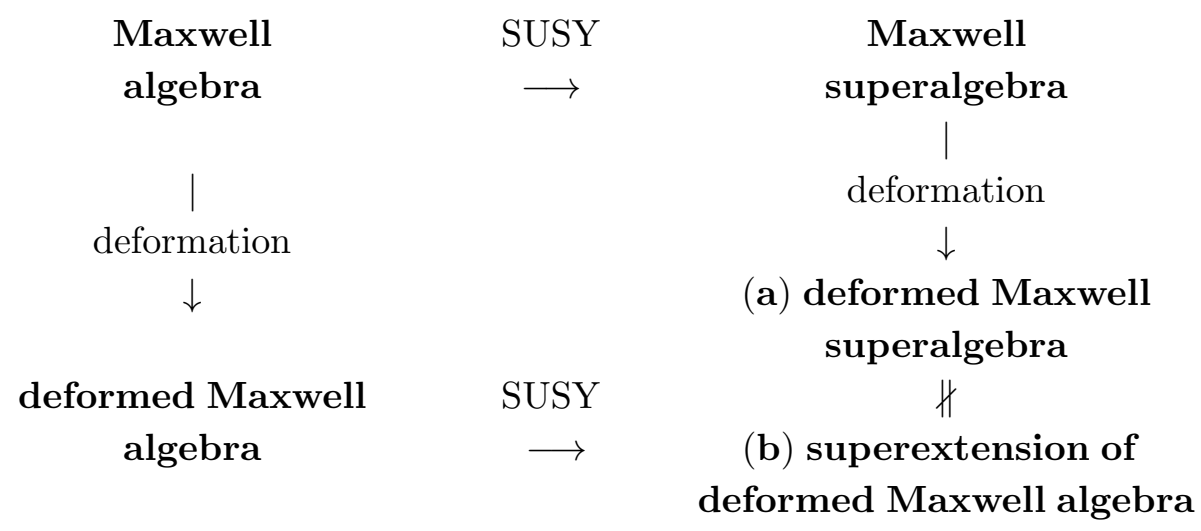

Fig. 1 Two ways of modifying the Maxwell algebra

We are following the upper path leading to (a) in Fig. 1, on the other hand Soroka and Soroka [22] recently have chosen the other path leading to (b). The results do not coincide, because the supersymmetrization of Maxwell algebra requires eight real supercharges while that of the deformed Maxwell algebra, $O(3,1) \oplus O(3,2) \rightarrow O(3,1) \oplus O S p(1 \mid 4)$, only needs four real supercharges.

It is plausible to assume, by analogy with the $\mathrm{N}=1$ case considered in this paper, that the N-extended Maxwell superalgebra can be obtained by suitable contraction of the $O S p(2 N \mid 4)$ algebra $^{6}$ and it will have $8 \mathrm{~N}$ real supercharges. We conjecture as well that the D-dimensional Maxwell superalgebra can be obtained by contraction of the Ddimensional AdS superalgebra, i.e., it exists in those dimensions $(\mathrm{D}=2,3,4,5,7)$ in which we can formulate AdS superalgebras ${ }^{7}$ [30]. An interesting question is whether it is possible to construct Maxwell superalgebras with bosonic Maxwell subalgebra in other dimensions, e.g., in $\mathrm{D}=10$ and $\mathrm{D}=11$.

Our considerations above are only valid if we assume that the supersymmetrization of Maxwell algebra, which can be called minimal, satisfies the following two assumptions:

1. it is an enlargement of Poincarè superalgebra with the four-momenta present in the anticommutator of supercharges,

\footnotetext{
${ }^{6}$ In principle one can obtain the same algebra by contraction of the $\mathrm{N}$-extended $\mathrm{D}=4 \mathrm{dS}$ superalgebra, but algebraically this procedure is more complicated due to the presence of quaternionic Majorana subsidiary conditions.

${ }^{7}$ One can also obtain the D-dimensional Maxwell superalgebra by contracting D-dimensional dS superalgebras, but dS superalgebras exist only for $\mathrm{D}=2,3,4,5$ (see also Appendix B).
} 
2. its bosonic sector is the direct sum of Maxwell algebra (1.1)-(1.3) and possibly some internal sector.

For if we allow generalized Maxwell superalgebras, for example, with the following basic SUSY relation $\{Q, Q\}=b Z$ (see [5]) that contradicts our first assumption, or with a bosonic sector that is a non-Abelian enlargement of Maxwell algebra, we open up a "Pandora's box" of possibilities. The simplest Abelian enlargement consists in adding to Maxwell algebra some central charges, like $B$ and $B_{5}$ in $\mathrm{D}=4$ (see section 2), but one can also enlarge Maxwell algebra into a semisimple group with additional bosonic generators. One category of such extensions is described by the Bergshoeff-Sezgin superalgebra [21]. If we consider $\mathrm{D}=10,11$, which play a crucial role in string/M theory, one can look for contractions of generalized $\mathrm{D}=10$ and $\mathrm{D}=11$ AdS algebra $O S p(1 \mid 32)$, see for example [31], and look for the corresponding generalized $\mathrm{D}=10$ and $\mathrm{D}=11$ Maxwell superalgebras.

\section{Appendix}

\section{A. Notations and conventions}

Here we summarize our notations and conventions.

We use a positive signature space-time metric $\eta_{\mu \nu}=\operatorname{diag}(-,+, \ldots,+)$. Antisymmetrization is defined by

$$
A_{[\mu} B_{\nu]}=A_{\mu} B_{\nu}-A_{\nu} B_{\mu} .
$$

Our convention of hermitian conjugation for odd generators is $\mathbf{L}^{\dagger}=\mathbf{L}, \mathbf{Q}^{\dagger}=-\mathbf{Q}$, so that $\mathbf{Q}_{\alpha} \mathbf{L}^{\alpha}$ in the MC one-form $\Omega$ is Hermitian,

$$
\left(\mathbf{Q}_{\alpha} \mathbf{L}^{\alpha}\right)^{\dagger}=\mathbf{L}^{\alpha \dagger} \mathbf{Q}_{\alpha}^{\dagger}=\mathbf{L}^{\alpha}\left(-\mathbf{Q}_{\alpha}\right)=\left(\mathbf{Q}_{\alpha} \mathbf{L}^{\alpha}\right) .
$$

The conjugate for Majorana spinors $\overline{\mathbf{L}}$ 's are defined using charge conjugation matrix $C$ as

$$
\overline{\mathbf{L}}_{\alpha}=\mathbf{L}^{\beta} C_{\beta \alpha} .
$$

In 4 dimensions we use the gamma matrices in the Majorana representation

$$
\gamma_{0}=i \sigma_{2} \otimes I_{2}, \quad \gamma_{1}=\sigma_{3} \otimes \sigma_{1}, \quad \gamma_{2}=\sigma_{3} \otimes \sigma_{3}, \quad \gamma_{3}=\sigma_{1} \otimes I_{2} .
$$

They are all real and verify the Clifford algebra

$$
\left\{\gamma_{\mu}, \gamma_{\nu}\right\}=2 \eta_{\mu \nu}, \quad \eta_{\mu \nu}=(-,+++), \quad \mu, \nu=0,1,2,3
$$

The $O(3,1)$ Lorentz generators are $\gamma_{\mu \nu}=\frac{1}{2}\left[\gamma_{\mu}, \gamma_{\nu}\right]=-\gamma_{\nu \mu}$ and satisfy

$$
\left[\gamma_{\mu \nu}, \gamma_{\rho \sigma}\right]=2\left(\eta_{\rho[\nu} \gamma_{\mu] \sigma}-\eta_{\sigma[\nu} \gamma_{\mu] \rho}\right)
$$

The charge conjugation matrix $C$ and $\gamma_{5}$ are also real

$$
C=\gamma_{0}, \quad \gamma_{5}=\gamma_{0123}=\sigma_{1} \otimes i \sigma_{2}
$$


and satisfy

$$
C^{T}=-C=C^{-1}, \quad \gamma_{\mu}^{T}=C \gamma_{\mu} C^{-1}
$$

then

$$
\left(C \gamma_{\mu}\right)^{T}=-\left(C \gamma_{\mu}\right), \quad\left(C \gamma_{\mu \nu}\right)^{T}=\left(C \gamma_{\mu \nu}\right), \quad\left(C \gamma_{5}\right)^{T}=-\left(C \gamma_{5}\right) .
$$

The cyclic identities necessary for the closure of the superalgebras are

$$
\left(C \gamma_{\mu}\right)_{(\alpha \beta}\left(C \gamma^{\mu}\right)_{\gamma \delta)}=0, \quad(\alpha \beta \gamma \delta) \text { symmetric sum. }
$$

The $O(3,2)$ gamma matrices $\Gamma_{\hat{\mu}}$ 's are expressed by using the Majorana representation of the $O(3,1)$ gamma matrices (A.4) as

$$
\Gamma_{\mu}=\gamma_{\mu} \gamma_{5}, \quad \Gamma_{4}=-\gamma_{5},
$$

and verify the $O(3,2)$ Clifford algebra

$$
\left\{\Gamma_{\hat{\mu}}, \Gamma_{\hat{\nu}}\right\}=2 \eta_{\hat{\mu} \hat{\nu}}, \quad \eta_{\hat{\mu} \hat{\nu}}=(-,+++;-), \quad \hat{\mu}, \hat{\nu}=0,1,2,3,4 .
$$

The $O(3,2)$ generators $\Gamma_{\hat{\mu} \hat{\nu}}$ are

$$
\Gamma_{\mu \nu}=\frac{1}{2}\left[\Gamma_{\mu}, \Gamma_{\nu}\right]=\gamma_{\mu \nu}, \quad \Gamma_{\mu 4}=\Gamma_{\mu} \Gamma_{4}=\gamma_{\mu},
$$

and satisfy

$$
\left[\Gamma_{\hat{\mu} \hat{\nu}}, \Gamma_{\hat{\rho} \hat{\sigma}}\right]=2\left(\eta_{\hat{\rho}[\hat{\nu}} \Gamma_{\hat{\mu}] \hat{\sigma}}-\eta_{\hat{\sigma}[\hat{\nu}} \Gamma_{\hat{\mu}] \hat{\rho}}\right) .
$$

It gives the real Majorana representation of the algebra (A.12) with the charge conjugation matrix $C=\gamma_{0}=-\Gamma_{0} \Gamma_{4}$,

$$
(C)^{T}=-(C), \quad\left(C \Gamma_{\hat{\mu}}\right)^{T}=-\left(C \Gamma_{\hat{\mu}}\right), \quad\left(C \Gamma_{\hat{\mu} \hat{\nu}}\right)^{T}=\left(C \Gamma_{\hat{\mu} \hat{\nu}}\right) .
$$

The $O(4,1)$ gamma matrices $\Gamma_{\hat{\mu}}$ are

$$
\Gamma_{\mu}=\gamma_{\mu} \gamma_{5}, \quad \Gamma_{4}=i \gamma_{5}
$$

They verify the $O(4,1)$ Clifford algebra

$$
\left\{\Gamma_{\hat{\mu}}, \Gamma_{\hat{\nu}}\right\}=2 \eta_{\hat{\mu} \hat{\nu}}, \quad \eta_{\hat{\mu} \hat{\nu}}=(-,+++;+), \quad \hat{\mu}, \hat{\nu}=0,1,2,3,4 .
$$

The $O(4,1)$ generators $\Gamma_{\hat{\mu} \hat{\nu}}$ are

$$
\Gamma_{\mu \nu}=\frac{1}{2}\left[\Gamma_{\mu}, \Gamma_{\nu}\right]=\gamma_{\mu \nu}, \quad \Gamma_{\mu 4}=\Gamma_{\mu} \Gamma_{4}=-i \gamma_{\mu},
$$

where

$$
\left[\Gamma_{\hat{\mu} \hat{\nu}}, \Gamma_{\hat{\rho} \hat{\sigma}}\right]=2\left(\eta_{\hat{\rho}[\hat{\nu}} \Gamma_{\hat{\mu}] \hat{\sigma}}-\eta_{\hat{\sigma}[\hat{\nu}} \Gamma_{\hat{\mu}] \hat{\rho}}\right) .
$$

Using the same charge conjugation matrix $C=\gamma_{0}=i \Gamma_{0} \Gamma_{4}$, we find that the same relations A.15) hold also for $O(4,1)$ gamma matrices. In contrast to the AdS case these gamma matrices are complex but satisfy

$$
\Gamma_{\hat{\mu}}^{*}=-\Gamma_{4} \Gamma_{\hat{\mu}} \Gamma_{4}, \quad \Gamma_{\hat{\mu} \hat{\nu}}^{*}=\Gamma_{4} \Gamma_{\hat{\mu} \hat{\nu}} \Gamma_{4} .
$$




\section{B. Quaternionic (super)groups and (super)algebras}

\section{B.1 Quaternionic algebras}

Quaternions $q$, the elements of quaternionic space $H^{n}=\left(q_{1} \ldots q_{n}\right)$, are written using

$$
q=q^{(0)}+e_{r} q^{(r)} \in H, \quad q^{(0)}, q^{(r)} \in R,
$$

where the quaternionic units $e_{r}$ 's satisfy the multiplication rule

$$
e_{r} e_{s}=-\delta_{r s}+\epsilon_{r s t} e_{t}
$$

Quaternionic conjugate $\bar{q}$ of $q$ is defined by $\bar{q}=q^{(0)}-e_{r} q^{(r)}$. Remember $q-\bar{q}=0, \rightarrow q^{(r)}=$ $0,(r=1,2,3), q+\bar{q}=0, \rightarrow q^{(0)}=0$ and it holds $\overline{p q}=\bar{q} \bar{p}$.

\section{B.2 Quaternionic groups and corresponding Lie algebras [32] [33]}

i) The pseudounitary quaternionic (unitary) group $U(n-k, k ; H)$;

It consists of the generators of linear quaternionic $n \times n$ matrix transformations preserving the Hermitian scalar product

$$
\langle\bar{q} \mid p\rangle=\sum_{i=1}^{n-k} \bar{q}_{i} p_{i}-\sum_{j=1}^{k} \bar{q}_{n-k+j} p_{n-k+j} \equiv \sum_{K, L=1}^{n} \bar{q}_{K} \eta_{K L} p_{L} .
$$

The transformation of $q$ and $p$ is

$$
p_{K} \rightarrow U_{K L} p_{L}, \quad q_{K} \rightarrow U_{K L} q_{L}, \quad \bar{q}_{K} \rightarrow \bar{q}_{L} \overline{U_{K L}} \equiv \bar{q}_{L} U_{L K}^{\dagger}
$$

where $\dagger$ is defined using quaternionic conjugation. Then the condition of invariance of the metric is

$$
U^{\dagger} \eta U=\eta, \quad \rightarrow \quad \eta_{K N} \overline{U_{K L}} U_{N M}=\eta_{L M}
$$

Number of independent conditions (in real components) following from (B.5) are $4 \frac{n(n-1)}{2}$ for off-diagonal and $n$ for diagonal components, i.e., the number of independent real group parameters is $4 n^{2}-4 \frac{n(n-1)}{2}-n=n(2 n+1)$. For arbitrary $n, k$ the norm (B.3) defines the pseudounitary quaternionic (unitary) group $U(n-k, k ; H)$.

ii) The antiunitary quaternionic group $U_{\alpha}(n ; H)$

One can define as well in quaternionic space the antiunitary quaternionic group $U_{\alpha}(n ; H)$ as preserving the antihermitian quaternionic scalar product [33] 17]

$$
\langle\bar{q} \mid p\rangle_{A}=\sum_{K, L=1}^{n} \bar{q}_{K} A_{K L} p_{L}
$$

with

$$
A_{K L}=-\bar{A}_{L K}, \quad \rightarrow \quad A=-A^{\dagger}
$$


For even $n(n=2 k)$ one can choose $A_{K L}$ to be a real antisymmetric $2 k \times 2 k$ matrix, and for odd $n=2 k+1$ the last element $A_{2 k+1,2 k+1}$ should be purely quaternionic (one usually assumes that it is given by the unit $e_{2}$ ). Then the condition of invariance of the metric is

$$
U^{\dagger} A U=A
$$

The number of independent conditions (in real components) following from (B.8) is $4 \frac{n(n-1)}{2}$ for off-diagonal and $3 n$ for diagonal components, i.e., the number of independent real group parameters is $4 n^{2}-4 \frac{n(n-1)}{2}-3 n=n(2 n-1)$. For arbitrary $n$ the norm (B.6) defines the antiunitary quaternionic group $U_{\alpha}(n ; H)$.

We could also consider orthogonal quaternionic groups $O(n ; H)$ and symplectic ones $S p(2 n ; H)$. These two quaternionic groups are defined as preserving the symmetric and antisymmetric scalar products respectively,

$$
\langle q \mid p\rangle_{S}=\sum_{K, L=1}^{n} q_{K}^{T} \eta^{K L} p_{L}, \quad\langle q \mid p\rangle_{A}=\sum_{K, L=1}^{n} q_{K}^{T} A^{K L} p_{L},
$$

where the transposed quaternions are described by partial inversion $q \rightarrow q^{T}=q^{(0)}+e_{3} q^{(3)}-$ $e_{2} q^{(2)}+e_{1} q^{(1) 8}$. However these Lie algebras are related to the unitary and antiunitary ones by the following relations,

$$
\begin{aligned}
& O(n ; H)=U_{\alpha}(n ; H), \quad n(2 n-1) \quad \text { real parameters } \\
& S p(2 n ; H)=U(n, n ; H), \quad 2 n(4 n+1) \quad \text { real parameters }
\end{aligned}
$$

as can be shown by using the complex realization of the quaternionic scalar products $(\overline{B .9})$.

iii) The complex representation of quaternions and quaternionic groups

In order to describe the quaternionic groups and algebras using complex variables, one can introduce the known representation of the quaternionic algebra (B.2) in terms of $2 \times 2$ Pauli matrices

$$
e_{r} \leftrightarrow-i\left(\sigma_{r}\right)_{i j}, \quad \rightarrow \quad q=\left(\begin{array}{cc}
q^{(0)}-i q^{(3)} & -q^{(2)}-i q^{(1)} \\
q^{(2)}-i q^{(1)} & q^{(0)}+i q^{(3)}
\end{array}\right)=\left(\begin{array}{cc}
z^{1} & -z^{2 *} \\
z^{2} & z^{1 *}
\end{array}\right) .
$$

Multiplication of quaternions is the product of their matrix representations. The conjugate $\bar{q}$ is described as the hermitian conjugate of the complex matrix $q$

$$
\bar{q}=q^{(0)}-i\left(\sigma_{r}\right)\left(-q^{(r)}\right), \quad \rightarrow \quad \bar{q}=\left(\begin{array}{cc}
q^{(0)}+i q^{(3)} & q^{(2)}+i q^{(1)} \\
-q^{(2)}+i q^{(1)} & q^{(0)}-i q^{(3)}
\end{array}\right)=\left(\begin{array}{cc}
z^{1 *} & z^{2 *} \\
-z^{2} & z^{1}
\end{array}\right)
$$

In this way we identify a quaternion with a pair of complex numbers,

$$
q \leftrightarrow\left(\begin{array}{c}
z^{1} \\
z^{2}
\end{array}\right), \quad q \in H, \quad\left(\begin{array}{c}
z^{1} \\
z^{2}
\end{array}\right) \in C^{2} .
$$

\footnotetext{
${ }^{8}$ The way the transposed quaternions are defined follows from the complex realization (B.11). It should be recalled that the standard framework for the description of all Lie algebras are complex algebras, and the quaternionic variables are rather used as auxiliary notation.
} 
The components of the second column of $q$ are determined from those of the first.

Using the substitutions (B.11), (B.12), one can express the quaternionic scalar product (B.3) as pairs of complex numbers, namely ${ }^{9}$ 32 34

$$
\langle\bar{q} \mid p\rangle_{S}=\left(\begin{array}{c}
z_{A}^{1 *} \eta_{A B} u_{B}^{1}+z_{A}^{2 *} \eta_{A B} u_{B}^{2} \\
z_{A}^{1} \eta_{A B} u_{B}^{2}-z_{A}^{2} \eta_{A B} u_{B}^{1}
\end{array}\right)=\left(\begin{array}{c}
\left(z_{A}^{1 *}, z_{A}^{2 *}\right)\left(\begin{array}{cc}
\eta_{A B} & 0 \\
0 & \eta_{A B}
\end{array}\right)\left(\begin{array}{l}
u_{B}^{1} \\
u_{B}^{2}
\end{array}\right) \\
\left(z_{A}^{1}, z_{A}^{2}\right)\left(\begin{array}{cc}
0 & \eta_{A B} \\
-\eta_{A B} & 0
\end{array}\right)\left(\begin{array}{l}
u_{B}^{1} \\
u_{B}^{2}
\end{array}\right)
\end{array}\right) .
$$

Invariant transformations of the inner product are those of the $2 n$ complex vectors that keep the two components of (B.14) invariant. It follows that in complex $2 n$ dimensional space $\left(z_{A}^{1}, z_{A}^{2}\right) \in C^{2 n}$ one can introduce a pair of complex scalar products; first describing $U(2 n-2 k, 2 k ; C)$ with diagonal pseudo-metric $\tilde{\eta}=\left(\begin{array}{cc}\eta_{A B} & 0 \\ 0 & \eta_{A B}\end{array}\right)$ and second with $2 n \times 2 n$ antisymmetric metric $\tilde{A}=\left(\begin{array}{cc}0 & \eta_{A B} \\ -\eta_{A B} & 0\end{array}\right)$ describing the group $S p(2 n ; C)$. One obtains in complex notation the following equivalence as follows

$$
U(n-k, k ; H)=U(2 n-2 k, 2 k ; C) \cap S p(2 n ; C)=U S p(2 n-2 k, 2 k ; C) .
$$

For the antihermitian quaternionic inner product $(\mathrm{B} .6)$ the complex components are written as

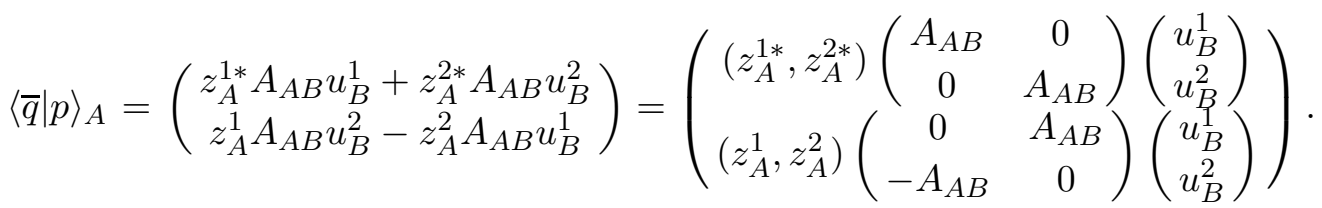

Here the symmetric $2 n \times 2 n$ matrix $\hat{\eta}=\left(\begin{array}{cc}0 & A_{A B} \\ -A_{A B} & 0\end{array}\right)$ has the signature $(1, \ldots, 1,-1, \ldots,-1)$ and defines the complex group $O(n, n ; C)=O(2 n ; C)^{10}$ and the antisymmetric $\hat{A}=$ $\left(\begin{array}{cc}A_{A B} & 0 \\ 0 & A_{A B}\end{array}\right)$ defines the antiunitary complex group $S p(2 n ; C)$ which is equivalent with $U(n, n ; C)$, 33] 35. Then we obtain

$$
U_{\alpha}(n ; H)=O(2 n ; C) \cap U(n, n ; C)=O^{*}(2 n) .
$$

\section{B.3 Quaternionic supergroups and applications to space-time symmetries}

There exists only one infinite series of norm-preserving supergroups $U U_{\alpha}(n-k, k \mid m ; H)$ which leave invariant the following graded metric in quaternionic superspace $H^{n \mid m}$ spanned by the graded quaternionic vectors $\left(q_{1} \ldots q_{n} ; \theta_{1} \ldots \theta_{m}\right)$

$$
\langle\mathcal{Q} \mid \mathcal{P}\rangle=\sum_{K, L=1}^{n} \bar{q}_{K} \eta_{K L} p_{L}+\sum_{\alpha, \beta=1}^{m} \bar{\theta}_{\alpha} A_{\alpha \beta} \theta_{\beta}
$$

\footnotetext{
${ }^{9}$ The complex variables $u^{k}$ are related to the components of $p$ as $\left(z^{1}, z^{2}\right)$ are to those of $q-$ see (B.11).

${ }^{10}$ The orthogonal complex algebras do not have signatures $-O(n ; C)$ is the complexification of real algebras $O(n-k, k)$ for $k=0,1 \ldots n$.
} 
The variables $\theta_{\alpha}=\theta_{\alpha}^{(0)}+\theta_{\alpha}^{(r)} e_{r}$ are quaternions with Grassmann-valued components, i.e., $\theta_{\alpha}^{(0)}, \theta_{\alpha}^{(r)}$ form the basis of a real $4 m$-dimensional Grassmann algebra. The bosonic sector of the supergroup $U U_{\alpha}(n-k, k \mid m ; H)$ is given by the product of quaternionic groups $U(n-k, k ; H) \otimes U_{\alpha}(m ; H)$.

The quaternionic graded scalar product $(\mathrm{B} .18)$ can be expressed equivalently by a pair of complex graded scalar products defining the pair of complex supergroups $U(2 n-$ $2 k, 2 k \mid m, m)$ and $O S p(2 m \mid 2 n ; C)$. We have the following equivalence in quaternionic and complex notation [37] [34]

$$
U U_{\alpha}(n-k, k \mid m ; H)=U(2 n-2 k, 2 k \mid m, m) \cap O S p(2 m \mid 2 n ; C) .
$$

In the description of space-time symmetries the quaternionic groups are given as

$$
\overline{O(4,1)}=U(1,1 ; H)=U S p(2,2)
$$

and for $\mathrm{D}=7$ anti-de-Sitter symmetries [32] as

$$
\overline{O(6,2)}=U_{\alpha}(4 ; H)=O^{*}(8) \text {. }
$$

The $n$-extended $\mathrm{D}=4 \mathrm{dS}$ supergroup is [17]- 19$]$

$$
U U_{\alpha}(1,1 \mid n ; H)=U(2,2 \mid n, n) \cap O S p(2 n \mid 4 ; C)
$$

with internal symmetries $U(n ; H)=U S p(2 n)$, and the $\mathrm{D}=7$ AdS supergroup, after using the relation $U(n \mid m)=U(m \mid n)$ valid for graded unitary supergroups, takes the form [17] 335

$$
U_{\alpha} U(4 \mid n ; H)=U(4,4 \mid 2 n) \cap O S p(2 n \mid 8 ; C) .
$$

The internal symmetries in $\mathrm{D}=7$ are given by $U_{\alpha}(n ; H)=O^{*}(2 n)$.

\section{B.4 D=4 dS superalgebra}

The graded de-Sitter algebras in $\mathrm{D}=2,3,4,5$ are the same as $\mathrm{D}=3,4,5,6$ Lorentz algebras. They are described by a symplectic series of real, complex or quaternionic Lie algebras $S p(2, F),(F=R, C, H)$ and quaternionic $S l(2, H)^{11}$

$$
\begin{array}{ll}
D=2: & s p(2 ; R)=s l(2 ; R)=o(2,1) \\
D=3: & s p(2 ; C)=s l(2 ; C)=o(3,1) \\
D=4: & s p(2 ; H)=u(1,1 ; H)=o(4,1) \\
D=5: & s l(2 ; H)=s u^{*}(4)=o(5,1) .
\end{array}
$$

If we employ the relations (B.24) as linking various groups, we obtain the series of double spinorial coverings of $O(D, 1)$ for $\mathrm{D}=2,3,4,5$ :

$$
\begin{aligned}
& \overline{O(2,1)}=S L(2 ; R) \\
& \overline{O(3,1)}=S L(2 ; C) \\
& \overline{O(4,1)}=U(1,1, H) \\
& \overline{O(5,1)}=S L(2, H) .
\end{aligned}
$$

\footnotetext{
${ }^{11}$ In the third relation in $(B .24)$ we have used the second of $(B .10)$.
} 
The supersymmetrization of space-time symmetries requires the knowledge of the fundamental spinor representation of the corresponding spinorial covering group. We shall assume that the (super)algebra is supersymmetrized by the introduction of supercharges, which transform under the transformations of space-time symmetry group as the fundamental spinor representation. ${ }^{12}$

The spinorial coverings $(\mathrm{B} .25)$ are supersymmetrized as follows:

$$
\begin{aligned}
& D=2: \quad O S p(N \mid 2 ; R) \text { or } S L(2 \mid N ; R) \\
& D=3: \quad S L(2 \mid N ; C) \\
& D=4: \quad U U_{\alpha}(1,1 \mid N ; H) \\
& D=5: \quad S L(2 \mid N ; H) \text {. }
\end{aligned}
$$

The $\mathrm{N}=1, \mathrm{D}=4 \mathrm{dS}$ superalgebra $U U_{\alpha}(1,1 \mid 1 ; H)$ used in this paper can be translated into complex notation by using the relations (B.11), (B.12), but this is quite a tedious procedure (see for example the complex description of $\operatorname{sl}(2 \mid N ; H)$ in [34]). In this paper we obtained the $\mathrm{N}=1, \mathrm{D}=4 \mathrm{dS}$ superalgebra (see (3.25)) by observing its correspondence with the well-known $\mathrm{N}=2, \mathrm{D}=4$ AdS superalgebra (see (3.18)). Such a procedure follows from the property that $O S p(2 \mid 4 ; R)$ as well as $U U_{\alpha}(1,1 \mid 1 ; H)$ are two different real forms of $O S p(2 \mid 4 ; C)$ and consists of the following steps:

1. Replace the real $4 \times 4$ matrices (A.11) describing $O(3,2)$ Dirac matrices by the complex $4 \times 4$ matrices $\left(\right.$ A.16) giving the $O(4,1)$ Dirac algebra. ${ }^{13}$

2. The choice of $O(4,1)$ gamma matrices dictates the choice of $O(4,1)$ conjugation matrix $C$, satisfying the defining relations (A.15) for $O(4,1)$ Dirac matrices. In our paper we select the same explicit form of $C$-matrix for $O(3,2)$ and $O(4,1)$ (see appendix A).

3. Replace the $\mathrm{N}=2$ real $O S p(2 \mid 4)$ supercharges by complexified supercharges describing the complex-holomorphic superalgebra $O S p(2 \mid 4 ; C)$.

4. Impose invariance of the superalgebra $O S p(2 \mid 4 ; C)$ under the quaternionic Majorana conjugation implying that the supercharges are $O(4,1)$ quaternionic spinors

$$
\mathcal{Q}_{\alpha} \rightarrow a \epsilon^{i j}\left(\Gamma_{4}\right)_{\alpha}{ }^{\beta} \mathcal{Q}_{\beta j}^{\dagger}
$$

where $|a|=1$ due to the involutive character of the mapping (B.27) (in (3.26) the choice $a=i$ is made).

Our superalgebra (3.25) satisfies all these requirements characterizing the $U U_{\alpha}(1,1 \mid 1 ; H)$ superalgebra, namely

- It is covariant under $U(1,1 ; H) \otimes U_{\alpha}(1 ; H)=U S p(2,2) \otimes O(2)$ transformations.

\footnotetext{
${ }^{12}$ By this assumption we exclude so-called vector-supersymmetries [29].

${ }^{13}$ The complexification in our paper is obtained simply by multiplying the fifth real $O(3,2)$ gamma matrix by the imaginary unit $i$.
} 
- The 8 complex charges do satisfy the quaternionic Majorana condition (3.26).

In conclusion, we would like to add that $\mathrm{N}=1, \mathrm{D}=4 \mathrm{dS}$ supergravity, as a gauge theory on $U U_{\alpha}(1,1 \mid 1 ; H)$, was constructed first in [38]. Unfortunately, it has been shown [19] 18] that such a theory necessarily contains ghost states.

\section{Acknowledgments}

We acknowledge discussions with Jose A. de Azcarraga, Jaume Gomis, Toine Van Proeyen. One of the authors (J.L.) would like to thank Professors Yan-Gang Miao and Mo-Lin Ge for their superb hospitality at Nankai University during the time of completion of this paper. We also acknowledge financial support from projects FPA2007-66665-C0201, 2009SGR502, Polish Ministry of Science and High Education grant NN202 318534 and Consolider CPANCSD2007-00042.

\section{References}

[1] H. Bacry, P. Combe and J. L. Richard, "Group-theoretical analysis of elementary particles in an external electromagnetic field. 1. The relativistic particle in a constant and uniform field," Nuovo Cim. A 67 (1970) 267.

[2] R. Schrader, "The Maxwell group and the quantum theory of particles in classical homogeneous electromagnetic fields," Fortsch. Phys. 20 (1972) 701.

[3] J. Beckers and V. Hussin, "Minimal electromagnetic coupling schemes. II. Relativistic and nonrelativistic Maxwell groups," J. Math. Phys. 24 (1983) 1295.

[4] J. Negro and M. A. del Olmo "Local realizations of kinematical groups with a constant electromagnetic field. I. The relativistic case" J. Math. Phys. 31 (1990) 568.

[5] D. V. Soroka and V. A. Soroka, "Tensor extension of the Poincaré algebra," Phys. Lett. B 607 (2005) 302 [arXiv:hep-th/0410012].

[6] S. Bonanos and J. Gomis, "A note on the Chevalley-Eilenberg cohomology for the Galilei and Poincaré algebras," J. Phys. A: Math. Theor. 42 (2009) 145206 [arXiv:0808.2243[hep-th]].

[7] S. Bonanos and J. Gomis, "Infinite sequence of Poincaré group extensions: structure and dynamics," J. Phys. A: Math. Theor. 43 (2010) 015201 [arXiv:0812.4140 [hep-th]].

[8] J. Gomis, K. Kamimura and J. Lukierski, "Deformed Maxwell algebras and their realizations," JHEP 08 (2009) 039, [arXiv:0910.0326[hep-th]].

[9] G. W. Gibbons, J. Gomis and C. N. Pope, "Deforming the Maxwell-Sim algebra," [arXiv:0910.3220 [hep-th]].

[10] S. Bonanos, J. Gomis, K. Kamimura and J. Lukierski, "Maxwell superalgebra and superparticle in constant gauge backgrounds," Phys. Rev. Lett. 104 (2010) 090401 [arXiv:0911.5072 [hep-th]].

[11] D. V. Soroka and V. A. Soroka, "Semi-simple extension of the (super)Poincaré algebra," Adv. High Energy Phys. 10 (2009) 1155 [arXiv:hep-th/0605251].

[12] A. S. Galperin, E. A. Ivanov, V. I. Ogievetsky and E. Sokatchev, "Gauge field geometry from complex and harmonic analyticities. I. Kähler and self-dual Yang-Mills cases," Ann. Phys. 185 (1988) 1. 
[13] A. S. Galperin, E. A. Ivanov, V. I. Ogievetsky and E. Sokatchev, "Gauge field geometry from complex and harmonic analyticities. II. Hyper-Kähler case," Ann. Phys. 185 (1988) 22.

[14] J. A. de Azcarraga, J. M. Izquierdo, M. Picon and O. Varela, "Generating Lie and gauge free differential (super)algebras by expanding Maurer-Cartan forms and Chern-Simons supergravity," Nucl. Phys. B 662, 185 (2003) [arXiv:hep-th/0212347].

[15] D. Cangemi, "One formulation for both lineal gravities through a dimensional reduction," Phys. Lett. B 297 (1992) 261 [arXiv:gr-qc/9207004].

[16] C. Duval, Z. Horvath and P. A. Horvathy, "Chern-Simons gravity, based on a non-semisimple group," [arXiv:0807.0977 [hep-th]].

[17] T. Kugo and P. K. Townsend, "Supersymmetry and the division algebras," Nucl. Phys. B 221 (1983) 357.

[18] J. Lukierski and A. Nowicki, "All possible de Sitter superalgebras and the presence of ghosts," Phys. Lett. B 151 (1985) 382.

[19] K. Pilch, P. van Nieuwenhuizen and M. F. Sohnius, "De Sitter superalgebras and supergravity," Commun. Math. Phys. 98 (1985) 105.

[20] G. W. Gibbons, J. Gomis and C. N. Pope, "General very special relativity is Finsler geometry," Phys. Rev. D 76 (2007) 081701 [arXiv:0707.2174 [hep-th]].

[21] E. Bergshoeff and E. Sezgin, "Super-p-brane theories and new space-time superalgebras," Phys. Lett. B 354 (1995) 256 [arXiv:hep-th/9504140].

[22] D. V. Soroka and V. A. Soroka, "Semi-simple $o(N)$-extended super-Poincaré algebra," [arXiv:1004.3194 [hep-th]].

[23] M. B. Green, "Supertranslations, superstrings and Chern-Simons forms," Phys. Lett. B 223 (1989) 157.

[24] M. Levy-Nahas, "Deformation and contraction of Lie algebras", J. Math. Phys. 8 (1967) 1211.

[25] S. Bonanos. http://www.inp.demokritos.gr/ sbonano/superEDC/.

[26] A. Van Proeyen, "Tools for supersymmetry," [arXiv:hep-th/9910030].

[27] P. D. Jarvis and H. S. Green, "Casimir invariants and characteristic identities for generators of the general linear, special linear and orthosymplectic graded Lie algebras," J. Math. Phys. 20 (1979) 2115.

[28] S. Bonanos and K. Kamimura, "On the Cayley-Hamilton theorem for supermatrices," [arXiv:1003.2667 [math-ph]].

[29] R. Casalbuoni, F. Elmetti, J. Gomis, K. Kamimura, L. Tamassia and A. Van Proeyen, "Vector supersymmetry: Casimir operators and contraction from $\operatorname{OSp}(3,2 \mid 2)$," JHEP 0901 (2009) 035 [arXiv:0812.1982 [hep-th]].

[30] J. W. van Holten and A. Van Proeyen, " $N=1$ supersymmetry algebras in $\mathrm{D}=2, \mathrm{D}=3, \mathrm{D}=4$ mod-8," J. Phys. A 15 (1982) 3763.

[31] E. Bergshoeff and A. Van Proeyen, "The many faces of OSp(1|32)," Class. Quant. Grav. 17 (2000) 3277 [arXiv:hep-th/0003261]. 
[32] R.Gilmore, "Lie groups, Lie algebras and some of their applications", Wiley \& Sons, New York, 1974.

[33] J.Tits, "Tabellen zu den einfachen Lie gruppen and ihre darstellungen", Lect. Notes in Math. Vol.40, Springer Verlag Berlin/Heidelberg/New York, 1967.

[34] J. Lukierski and A. Nowicki, "Quaternionic supergroups And D = 4 Euclidean extended supersymmetries," Annals Phys. 166 (1986) 164.

[35] Z. Hasiewicz, P. Morawiec and J. Lukierski, "Seven-dimensional de Sitter and six-dimensional conformal supersymmetries," Phys. Lett. B 130 (1983) 55.

[36] F. Gursey and H. C. Tze, "Complex and quaternionic analyticity in chiral and gauge theories. Part 1," Annals Phys. 128 (1980) 29.

[37] V. G. Kac, "A sketch of Lie superalgebra theory," Commun. Math. Phys. 53 (1977) 31.

[38] J. Lukierski and A. Nowicki, "Supersymmetry in the presence of positive cosmological constant," Univ.Wroclaw preprint No 609 (1984). 